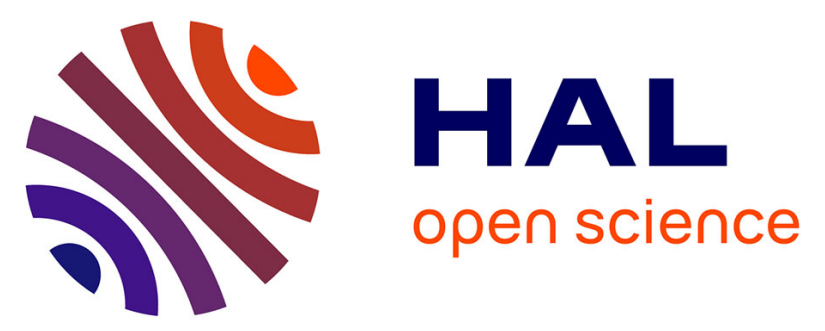

\title{
Recommandations pour l'évaluation et la prise en charge de la maladie de Lapeyronie : rapport du comité d'andrologie et de médecine sexuelle de l'AFU
}

Ludovic Ferretti, François-Xavier Madec, William Akakpo, Charlotte Methorst, Damien Carnicelli, Jean-Etienne Terrier, N. Morel Journel, Sebastien Beley, J.-P. Pierre Graziana, François Marcelli, et al.

\section{To cite this version:}

Ludovic Ferretti, François-Xavier Madec, William Akakpo, Charlotte Methorst, Damien Carnicelli, et al.. Recommandations pour l'évaluation et la prise en charge de la maladie de Lapeyronie: rapport du comité d'andrologie et de médecine sexuelle de l'AFU. Progrès en Urologie, 2021, 31 (8-9), pp.477-494. 10.1016/j.purol.2020.11.004 . hal-03525760

\section{HAL Id: hal-03525760 \\ https://hal.sorbonne-universite.fr/hal-03525760}

Submitted on 14 Jan 2022

HAL is a multi-disciplinary open access archive for the deposit and dissemination of scientific research documents, whether they are published or not. The documents may come from teaching and research institutions in France or abroad, or from public or private research centers.
L'archive ouverte pluridisciplinaire HAL, est destinée au dépôt et à la diffusion de documents scientifiques de niveau recherche, publiés ou non, émanant des établissements d'enseignement et de recherche français ou étrangers, des laboratoires publics ou privés. 


\section{Recommandations pour l'évaluation et la prise en charge de la maladie de Lapeyronie : rapport du comité d'andrologie et de médecine sexuelle de l'AFU}

Ludovic Ferretti $^{(1)}$, François-Xavier Madec ${ }^{(2)}$, William Akakpo ${ }^{(3)}$, Charlotte Methorst ${ }^{(4)}$ Damien Carnicelli ${ }^{(5)}$, Jean-Etienne Terrier ${ }^{(6)}$,Nicolas Morel Journel ${ }^{(5)}$, , Sebastien Beley ${ }^{(7)}$, Jean Pierre Graziana ${ }^{(8)}$, François Marcelli ${ }^{(9)}$,Vincent Hupertan ${ }^{(7)}$, René Yiou ${ }^{(10)}$, Kamel BenNaoum $^{(11)}$, Laurent Savareux ${ }^{(12)}$, Eric Huyghe ${ }^{(13)}$, Antoine Faix ${ }^{(14)}$

(1) : Service d'urologie, MSP Bordeaux Bagatelle, Talence

(2) : Service d'urologie, hôpital Foch, Suresnes

(3) : Département d'urologie, Hôpital Pitié-Salpétrière, Paris

(4) : Service d'urologie, hôpital des 4 Villes, Saint-Cloud

(5) : Service d'urologie, hôpital Privé Jean Mermoz, Lyon

(6) : Service d'urologie, Hôpital Lyon Sud, Pierre-Bénite

(7) : Clinique Turin, Paris

(8) : Clinique Mutualiste de la Porte de l'Orient, Lorient

(9) : Service d'urologie, andrologie et transplantation rénale, hôpital Huriez CHU Lille,

(10) : Département d'urologie, CHU Henri Mondor, Créteil

(11) : Service d'urologie, CHU Carémeau, Nîmes

(12) : Service d'urologie, Hôpital Privé la Chataigneraie, Beaumont

(13) : Département d'urologie, CHU Rangueil, Toulouse

(14) : Centre d'urologie du Polygone, Montpellier 


\section{Résumé :}

Introduction: La maladie de Lapeyronie est un motif fréquent de consultation en urologie, dont le traitement reste sujet à de nombreuses controverses. Elle n'a fait l'objet d'aucune recommandation française jusqu'à présent. Le Comité d'Andrologie et de Médecine Sexuelle de l'Association Française d'Urologie propose donc une série de recommandations basées sur les preuves.

Matériels et Méthodes : Ces recommandations sont réalisées selon la méthode ADAPTE, en se basant sur les recommandations européennes (EAU, ESSM), américaines (AUA, ISSM) et canadiennes (CAU), en intégrant les spécificités françaises en raison de la disponibilité des traitements, et une mise à jour de la bibliographie récente.

Résultats: L'évaluation de la maladie est clinique. Les patients présentant une gêne fonctionnelle ou un retentissement psychologique important peuvent se voir proposer un traitement. Les bénéfices et inconvénients de chaque traitement devront être explicités au patient. Concernant les traitements non chirurgicaux, aucun traitement disponible n'a l'autorisation de mise sur le marché en France. La vitamine E n'est pas recommandée. Des traitements à visée antalgiques (oraux ou ondes de choc de faible intensité) ou pro-érectiles peuvent être proposée selon le besoin, ainsi qu'une thérapie par traction. En raison de l'indisponibilité des injections de collagénase, les injections de vérapamil peuvent être proposées. Les traitements chirurgicaux sont à considérer en phase stabilisée de la maladie, et consistent en la réalisation d'une plicature, d'une incision-greffe ou de la pose d'un implant pénien en fonction du souhait du patient, de la courbure et de la taille de verge, ainsi que de la fonction érectile. Des traitements combinés peuvent être proposés.

Conclusion: La prise en charge de la maladie de Lapeyronie est complexe, et les niveaux de preuve des traitements sont faibles dans l'ensemble. Le succès du traitement dépendra de la qualité de l'évaluation initiale, de l'information du patient et de sa compréhension des effets attendus, et de l'expérience du praticien.

Mots clefs : maladie de Lapeyronie, recommandation, français, évaluation, traitement, médical, chirurgie, plicature, greffe, implant pénien 


\begin{abstract}
:
Introduction: Peyronie's disease is a common cause for consultation in urology. Many controversies surround its treatment. No French Guidelines have been published so far. The Committee of Andrology and Sexual Medicine of the French Association of Urology therefore offers a series of evidence-based recommendations.

Materials and Methods: These recommendations are made according to the ADAPTE method, based on European (EAU, ESSM), American (AUA, ISSM) and Canadian (CAU) recommendations, integrating French specificities due to the availability of treatments, and an update of the recent bibliography.

Results: The assessment of the disease is clinical. Patients with functional impairment or significant psychological repercussions may be offered treatment. The benefits and drawbacks of each treatment should be explained to the patient. Regarding non-surgical treatments, no available treatment has market authorization in France. Vitamin $\mathrm{E}$ is not recommended. Analgesic (oral or low-intensity shock waves) or proerectile treatments may be offered as needed, as well as traction therapy. Due to the unavailability of collagenase injections, verapamil injections may be offered. Surgical treatments are to be considered in the stabilized phase of the disease, and consist of performing a plication, an incision-graft or the placement of a penile implant according to the patient's wishes, the curvature and the penis size, as well as erectile function. Combination treatments can be offered.

Conclusion: The management of Peyronie's disease is complex, and the levels of evidence for treatments are generally low. The success of treatment will depend on the quality of the initial assessment, the patient's information and understanding of the expected effects, and the practitioner's experience.
\end{abstract}

Keywords : peyronie's disease, guideline, French, evaluation, treatment, medical, surgery, plication, graft, penile prosthesis 


\section{INTRODUCTION}

La maladie de Lapeyronie est une pathologie acquise de la tunique albuginée du corps caverneux caractérisée par la formation d'une plaque de fibrose, pouvant aboutir à des douleurs, une déformation de la verge en érection, et pouvant s'accompagner d'une dysfonction érectile. Elle fut décrite en 1743 par François-Gigot de Lapeyronie (1678-1747), premier chirurgien du roi Louis XV et fondateur de l'Académie Nationale de Chirurgie sous le terme «induratio plastica ». Cette pathologie décrite comme une maladie bénigne, peut impacter de façon importante la qualité de vie de l'homme et du partenaire.

Cette pathologie concerne l'homme le plus souvent dans la cinquième décade sur un terrain probablement prédisposant. Ce phénomène peut aboutir à une déformation de la verge (courbure, rétrécissement, étranglement, raccourcissement) et peut entraîner des difficultés de pénétration. Le stade précoce de la maladie de Lapeyronie est caractérisé par une réponse inflammatoire sous la tunique albuginée avec une prolifération fibroblastique formant une plaque rigide de tissus fibreux dans la tunique albuginée, pouvant évoluer vers une calcification voire une ossification [1].

\section{Objectif}

La littérature est pauvre en études épidémiologiques solides conduites dans une optique multicentrique utilisant une base de données conséquente et une analyse statistique rigoureuse.

C'est pourquoi le comité d'andrologie de l'AFU propose des recommandations françaises en se basant sur les recommandations existantes européennes, américaines et canadiennes [2-6] tout en les mettant à jour grâce aux dernières publications sur le sujet, selon la méthode ADAPTE.

\section{Epidémiologie}

Des études récentes semblent suggérer une prévalence jusqu'à $9 \%$, donc plus élevée qu'initialement décrite [7]. L'âge de survenue moyen semble être entre 55 et 60 ans. La douleur (parfois isolée) est présente dans plus de $50 \%$ des cas dans la phase inflammatoire, la déformation chez plus de $80 \%$, et la dysfonction érectile dans 20 à $50 \%$ des patients. Un traumatisme coïtal est retrouvé dans 5 à $13 \%$ des cas [8]. Des études épidémiologiques montrent également l'association avec le diabète, l'obésité, l'hypertension artérielle, l'hyperlipémie, le tabagisme et la chirurgie pelvienne, notamment après prostatectomie dans 15,9\% des cas. $[8,9]$.Elle peut être associée à une maladie de Dupuytren ainsi que d'autres pathologies du tissu conjonctif [1].

\section{Physiopathologie :}

La physiopathologie de cette affection reste mal connue, étant considérée comme multifactorielle. 
L'hypothèse du microtraumatisme albuginéal déclenchant est privilégiée. Les microhémorragies répétées entre les tuniques longitudinale externe et circulaire interne seraient à l'origine d'un afflux de Platelet Derived Growth Factor (PDGF) libéré par les plaquettes activées, et du Transforming Growth Factor $\beta 1$ (TGF $\beta 1$ ) [10-14]. L'activation du fibrinogène et le dépôt de fibrine seraient responsables de la migration des fibroblastes, colonisant ainsi l'architecture du squelette fibrineux. Il a été rapporté dans une étude immunohistochimique de la plaque la présence de $95 \%$ de fibrine, quasi inexistante dans l'albuginée saine $[15,16]$. On décrit aussi une augmentation de l'inhibiteur de l'activateur du plasminogène dans les plaques de fibrose, de l'inhibiteur de la fibrinolyse et de la collagénolyse [17].

L'action du TGF $\beta 1$ aboutit à la synthèse de tissu conjonctif, et à l'inhibition des collagénases. Cette molécule possède une capacité d'auto-régulation en amplifiant le phénomène [18-22].

Ces mécanismes sont à l'origine d'une dégradation des fibres élastiques dans $90 \%$ des cas et d'un dépôt anarchique de collagène de type 1, aboutissant à la plaque rétractile inextensible-pouvant être-responsable d'une maladie veino-occlusive locale [16,23].

Des prédispositions génétiques sont évoquées, tout comme une prédisposition aux soustypes HLA B27 et DR5.

\section{EVALUATION}

Le diagnostic de maladie de Lapeyronie est clinique, posé sur un faisceau d'arguments.

L'évaluation initiale a pour objectif de repérer les facteurs favorisants, préciser les symptômes, l'ancienneté des troubles, leur évolutivité ainsi que le retentissement psychologique sur le patient et le couple.

\section{Facteurs favorisants}

Les facteurs de risques éventuels à rechercher sont [9,24-28] :

- Antécédent de fracture ou traumatisme du pénis

- Antécédent de prostatectomie et chirurgie pelvienne

- Maladie de Dupuytren

- Maladie de Ledderhose

- Diabète

- Hypertension artérielle

- Dyslipidémie

- Cardiopathie ischémique

- Antécédent familial de maladie de Lapeyronie

- Tabagisme actif

- Ethylisme chronique 


\section{Phases de la maladie}

La phase aiguë (inflammatoire) est caractérisée par :

- La présence d'une douleur pénienne à l'état flaccide et/ou lors de l'érection

- L'apparition d'une déformation de la verge (courbure, rétrécissement, raccourcissement, étranglement) [29].

Ces symptômes sont inconstants. Cette phase est de durée variable, classiquement de 6 mois, mais parfois inexistante ou non perçue

La phase chronique est caractérisée par :

La régression de la douleur et l'installation de la déformation et le plus souvent l'apparition d'une plaque [14, 30]. Dans une étude portant sur l'histoire naturelle de la maladie de Lapeyronie chez 246 hommes, Mulhall et al. retrouvaient que 12\% des hommes avaient une amélioration spontanée, environ $40 \%$ restaient stables et jusqu'à $48 \%$ des hommes voyaient leur état s'aggraver avec le temps [14].

\section{Interrogatoire}

L'interrogatoire visera à rechercher la présence de douleur, d'une dysfonction érectile, de difficulté à la pénétration (faux pas du coït, instabilité pénienne, vécu de la partenaire), le type de sexualité afin de cibler au mieux les attentes du patient.

\section{Evaluation de la douleur}

L'évaluation de la douleur pourra être réalisée à l'aide d'échelle visuelle analogique (EVA), et sera utile dans le suivi évolutif de la maladie.

\section{Evaluation de la fonction érectile}

La recherche d'une dysfonction érectile, fréquemment associée à la maladie de Lapeyronie est une étape clé [31]. L'interrogatoire comporte une évaluation de la fonction érectile, avec l'aide eventuelle de questionnaires validés tels que l'International Index of Erectile Function (IIEF) ou l'EHS (Erection Hardness Score) [32].

L'interrogatoire précise si cette dysfonction érectile est antérieure, concomitante ou secondaire à la maladie de Lapeyronie. On recherche également les facteurs de risque classiques de la dysfonction érectile éventuellement associés [33].

\section{Evaluation psychologique}

La maladie de Lapeyronie peut avoir un impact négatif sur la qualité de vie et la santé mentale du patient et de sa partenaire. Une perte d'estime de soi ou de confiance, une anxiété de performance et/ou une dépression peuvent être associées d'emblée ou lors de l'évolution $[34,35,36]$. Les partenaires ont tendance à décrire une fonction sexuelle affectée et une baisse de la satisfaction pouvant également contribuer à la dysfonction érectile [36,37]. L'évaluation 
du/de la partenaire pourra être nécessaire en cas d'insatisfaction persistante.

\section{Examen du pénis et évaluation de la courbure}

Il se fait sur un pénis en traction, avec une palpation des corps caverneux de leur racine périnéale à l'extrémité distale glandulaire. L'examen recherche une ou plusieurs zones d'induration ou plaques fibreuses éventuellement douloureuses [3,31]. Afin de faciliter et rendre reproductible l'examen, une feuille d'examen standardisée pourra être utilisée (tableau $1)$.

Il n'existe pas de protocole standardisé de mesure de la longueur du pénis mais il est conseillé que le pénis soit mesuré sur sa face dorsale, étiré, depuis la base jusqu'au gland.

L'évaluation de la courbure pourra être réalisée à partir de photographies lors d'une érection physiologique ou après induction pharmacologique ou utilisation d'un vacuum en cas de difficulté à obtenir une érection de bonne qualité [38,39]. Idéalement, plusieurs angles de vue sont recommandés en position debout (Face, Profil, Vue du dessus) afin d'apprécier la déformation (figure 1).

\section{Questionnaire spécifique}

On peut s'aider éventuellement du Peyronie Disease's Questionnaire (PDQ). Ce questionnaire comporte 15 items mesurant l'impact et la sévérité des symptômes dans 3 domaines, à savoir les aspects psychologiques et physiques, la douleur et le score de gêne [40]. Ce questionnaire, reproductible, peut permettre de suivre l'évolution de la maladie dans le temps et après traitement, en particulier lors d'études cliniques.

\section{Examens paracliniques}

Le diagnostic de maladie de Lapeyronie est uniquement clinique.

Aucun examen complémentaire n'est obligatoire.

Un pharmaco-doppler pénien peut être utile, notamment en cas de dysfonction érectile associée ou avant intervention chirurgicale [41,42].

Un bilan biologique sera demandé en cas de dysfonction érectile avec facteurs de comorbidité et/ou baisse de la libido [43].

\section{Recommandations des sociétés savantes pour l'évaluation de la maladie de Lapeyronie}

ISSM (2016) Le diagnostic de maladie de Lapeyronie est clinique. L'évaluation de la courbure après injection intra caverneuse d'un agent vaso actif est recommandée avant traitement invasif.

AUA (2015) Le diagnostic de maladie de Lapeyronie est clinique. Une injection intra caverneuse de prostaglandines avec ou sans échographie doppler est recommandée avant traitement invasif. 
CAU (2018) Le diagnostic de maladie de Lapeyronie est clinique. Une injection intra caverneuse de prostaglandines est recommandée avant traitement invasif. L'échographie doppler couleur du pénis peut être utile pour la caractérisation d'anomalies de l'albuginée.

EAU (2019) Le diagnostic de maladie de Lapeyronie est clinique et l'échographie n'est recommandée que pour rechercher une cause vasculaire à la dysfonction érectile.

L'évaluation de la maladie de Lapeyronie constitue une recommandation de niveau 2 Grade B

Tableau 1 : Evaluation standardisée

\begin{tabular}{|c|c|c|}
\hline Interrogatoire & Examen du pénis au repos & $\begin{array}{l}\text { Examen du pénis en } \\
\text { érection }\end{array}$ \\
\hline $\begin{array}{l}\text { Recherche de facteurs } \\
\text { favorisants : maladie de } \\
\text { Dupuytren, traumatisme } \\
\text { génital, diabète, tabac, } \\
\text { antécédents urologiques }\end{array}$ & $\begin{array}{l}\text { Présence de } \begin{array}{r}\text { zones } \\
\text { d'induration, } \\
\text { localisation, taille }\end{array} \\
\end{array}$ & \\
\hline $\begin{array}{l}\text { Début et évolution des } \\
\text { symptômes : définir la phase } \\
\text { (inflammatoire } \\
\text { chronique) }\end{array}$ & Mesure du pénis en traction & $\begin{array}{l}\text { Déformation, angulation } \\
\text { (éventuel caractère } \\
\text { harmonieux), rétrécissement, } \\
\text { étranglement, encoche }\end{array}$ \\
\hline $\begin{array}{l}\text { Déformation du pénis : } \\
\text { angulation, rétrecissement, } \\
\text { étranglement, } \\
\text { raccourcissement }\end{array}$ & Gêne et douleur provoquée & $\begin{array}{l}\text { Sensibilité, } \\
\text { douloureuses }\end{array}$ \\
\hline Auto-palpation d'une plaque & & \\
\hline $\begin{array}{l}\text { Douleur pénienne: } \\
\text { localisation, circonstance } \\
\text { d'apparition (constante, lors } \\
\text { de l'érection) et évolution }\end{array}$ & & \\
\hline $\begin{array}{l}\text { Dysfonction érectile : } \\
\text { circonstance d'apparition } \\
\text { (préexistante aux symptômes } \\
\text { actuels ou synchrone), prise } \\
\text { médicamenteuse, mesure } \\
\text { objective (IIEF), forme } \\
\text { atypique de Dysfonction } \\
\text { érectile }\end{array}$ & & \\
\hline $\begin{array}{l}\text { Evaluation du retentissement } \\
\text { psychologique du patient et } \\
\text { de la partenaire }\end{array}$ & & \\
\hline
\end{tabular}

Figure 1 : angles de vue pour la prise de photographies en érection 


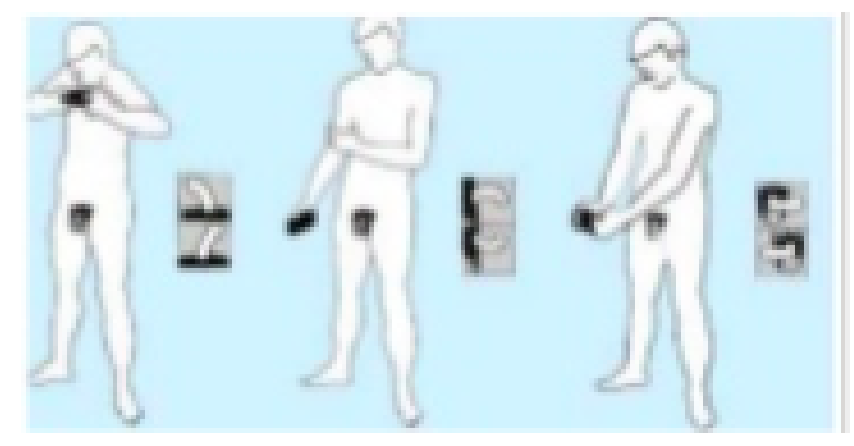

\title{
Annexes :
}

Annexe $1:$ IIEF-5 et EHS

Annexe 2 : PDQ (version française non validée)

\section{LES TRAITEMENTS NON CHIRURGICAUX}

\author{
LES TRAITEMENTS PAR VOIE ORALE
}

Les traitements oraux restent très fréquemment prescrits dans la prise en charge initiale de la MP[44]. Cependant, les données scientifiques pour soutenir leur utilisation sont limitées. La plupart de ces traitements ont été étudiés dans des essais cliniques de faible niveau de preuve, avec peu d'efficacité [2]. Aucun traitement oral n'a obtenu une AMM dans le cadre de la MP.

Avant toute prescription de ces traitements, il est nécessaire d'informer le patient des avantages et des risques associés à chacun de ces différents traitements oraux, mais aussi des autres options disponibles.

Les traitements oraux de la maladie de Lapeyronie peuvent être classés en trois catégories : les antalgiques, les anti-fibrotiques/antioxydants et les pros érectiles.

\section{Les traitements antalgiques par voie orale}

La douleur pénienne surtout présente pendant la phase active de la maladie doit être évaluée à l'aide d'une échelle visuelle analogique. En absence de contre-indications les antiinflammatoires non stéroïdiens par voie orale peuvent être prescrits pour une courte durée. Ce traitement doit être réévalué périodiquement $[6,46]$.

\section{Les traitements anti-fibrotiques et antioxydants.}

Le mécanisme d'action du potassium para-aminobenzoate (POTABA), décrit initialement in vitro en 1959, permet la diminution de la production de collagène dans des cultures de fibroblastes. 
Les effets de POTABA ont été étudiés dans une étude contrôlée contre placebo de 103 patients, qui a montré un taux de réponse de 74,3\% dans le groupe POTABA et de $50 \%$ dans le groupe placebo $(\mathrm{p}=0,016)$ sur la taille de la plaque avec stabilisation, sans efficacité sur la courbure ni sur la douleur [47].

\section{Devant les cas d'hépatites sévères voire léthales décrits, l'utilisation du POTABA n'est pas recommandée.}

\section{Vitamine E}

De nombreuses études ont évalué la vitamine E par voie orale dans le traitement de la ML, seule ou en association [48-52].

Une étude rétrospective, portant sur 31 patients n'a montré aucune différence sur la douleur, l'amélioration de la courbure, la réduction de la taille de la plaque ou l'amélioration de la fonction érectile entre les groupes vitamine E et contrôle [53].

Une étude prospective sur 30 patients n'a montré aucune différence entre trois groupes de traitement : vitamine E seule, IFNa2b intralésionnel seul ou association des deux [54].

Une étude plus vaste en double aveugle, randomisée et contrôlée portant sur 236 patients ne montre aucune amélioration significative sur la douleur, la courbure et la taille de la plaque entre les quatre groupes de traitement (placebo, vitamine E seule, propionyl-L-carnitine seule, association vitamine $\mathrm{E}$ et propionyl-L-carnitine), mais cette étude portait uniquement sur des patients stabilisés [55].

Un seul essai a démontré un effet thérapeutique de la vitamine E (dans le cadre d'un schéma thérapeutique combiné) avec une amélioration du score IIEF et une réduction de la taille des plaques $(\mathrm{P}<0,05)[56]$.

Par ailleurs il existe un doute quant aux risques cardiovasculaires en cas d'utilisation de la vitamine E à fortes doses et à long terme [57].

Devant l'ensemble de ces données, l'utilisation de la vitamine $E$ seule n'est pas recommandée dans la prise en charge de la ML.

\section{Colchicine}

La colchicine est un agent anti-microtubule qui réduit les dépôts de collagène et la fragmentation des fibres élastiques comme cela a été démontré chez le rat [58].

Les premières études non contrôlées sur de petites cohortes semblaient montrer une amélioration des symptômes et de la courbure [59,60].

Seuls 2 essais randomisés contre placebo ont été menés [51,61]. Le plus conséquent portait sur 84 patients et n'a lui aussi montré aucune différence significative entre la colchicine et le placebo en termes d'amélioration de la douleur, de la courbure du pénis ou de la taille de la plaque [61].

Par ailleurs, des effets indésirables fréquemment rapportés avec la colchicine

sont des troubles gastro-intestinaux, de la diarrhée, mais des événements plus graves, comme la myélosuppression ou une toxicité neuromusculaire[62].

L'utilisation de colchicine seule n'est pas recommandée dans la prise en charge de la ML.

\section{Tamoxifène}

Le tamoxifène agit en modulant de la sécrétion de TGF $\beta 1$ par les fibroblastes [63].

Sa première utilisation dans la ML date de 1992 sur une cohorte de 35 hommes. Les résultats ont montré une amélioration de la douleur, de la déformation et de la taille de la plaque[64]. 
Cependant un essai contrôlé randomisé chez 25 patients ne montre pas de différence significative entre les groupes sur la douleur, la courbure et la taille de la plaque [65].

L'utilisation du Tamoxifene n'est pas recommandée dans la prise en charge de la ML.

\section{Pentoxifylline}

La pentoxifylline est un inhibiteur non sélectif des phosphodiestérases permettant une accumulation de monoxyde d'azote, exerçant une activité antifibrotique en diminuant les concentrations de TGF $\beta$ [66].

Plusieurs centres ont publié des séries rétrospectives sans résultats significatifs[67].

Malgré des résultats prometteurs sur les cultures cellulaires et les modèles animaux, l'efficacité de la pentoxifylline n'a pas encore été démontrée chez les patients présentant une ML dans des études contrôlées. Le seul essai randomisé contrôlé contre placebo a été rétracté en raison de statistiques incohérences [68].

Cependant, une étude rapporte une stabilisation ou une diminution de la taille de la plaque sur une cohorte de 71 patients sans évaluation clinique de la douleur, de la déformation et de la fonction érectile [69].

Devant le faible niveau de preuve, l'utilisation de la pentoxifylline n'est pas recommandée.

\section{Les traitements pro-érectiles : Les IPDE 5}

Fiables, efficaces et d'une grande sécurité d'emploi les inhibiteurs de la 5-phospho diestérase (IPDE5) sont depuis longtemps le traitement de première ligne de la dysfonction érectile avec des taux d'efficacité, de satisfaction élevée en l'absence de contre-indication [70]. Cette comorbidité érectile est présente chez 30 à $45 \%$ des patients atteints par la ML [71].

Plusieurs études analysant des patients ayant une ML retrouvent une amélioration du score de fonction érectile (IIEF principalement) lorsqu'ils étaient traités par IPDE 5 [73-76].

En plus des effets bénéfiques sur la fonction érectile, il est probable que les IPDE-5 possèdent des propriétés anti-fibrotiques limitant la formation de la plaque [77].

Les IPDE 5 en prise quotidienne n'ont jamais prouvé leur efficacité dans le but d'améliorer la courbure [74,75]. Une étude contrôlée non randomisée portant sur 65 hommes atteints de cicatrices septales péniennes isolées sans déformation du pénis a été réalisée, avec 35 patients traités quotidiennement avec du Tadalafil $(2,5 \mathrm{mg})$. Une diminution de la cicatrice septale à l'échographie a été observée chez 24/35 patients (69\%) traités par le Tadalafil, contre $3 / 30(10 \%)$ dans le groupe contrôle [78].

Néanmoins, sur la base de l'efficacité et de la sécurité d'emploi des IPDE 5, de la forte prévalence de la dysfonction érectile chez les patients atteint de la ML et de l'effet potentiel anti-fibrotique, les IPDE -5 peuvent être proposés en cas de dysfonction érectile associée [79].

Recommandations des sociétés savantes pour le traitement oral de la maladie de Lapeyronie

ISSM (2016) Bénéfice faible ou nul des traitements oraux

AUA (2015) Les AINS peuvent être prescrits contre la douleur, mais la vitamine E, le tamoxifen, la procarbazine, les omega-3 ou une combinaison vitamine E-L carnitine ne doivent pas être proposée

CAU (2018) Pas d'effet prouvé, effets secondaires potentiels 
EAU (2019) Ne pas utiliser les traitements par vitamine E ou tamoxifen dans le but d'une réduction de la courbure. Les autres traitement médicaux (carnitine, pentoxifylline, colchicine) ne doivent pas être prescrits.

\section{Recommandation}

Le nombre et la qualité des études analysant l'efficacité des traitements oraux dans la maladie de Lapeyronie font défaut.

Néanmoins certains de ces traitements pourrait être prescrits à la phase active de la maladie ou chez des patients refusant les injections intra lésionnelles et les traitements chirurgicaux[80]. Ainsi, les traitements oraux présentant la meilleure balance bénéfice-risque sont les IPDE-5 en cas de DE associée, les AINS en cas de douleur péniennes au repos ou en érection.

Des travaux supplémentaires sont nécessaires, comprenant des essais randomisés contrôlés contre placebo sont afin de confirmer leur intérêt.

Le traitement par vitamine $E$ n'est pas recommandé.

\section{LES INJECTIONS INTRA-PLAQUE}

\section{Collagénase de Clostridium histolyticum (Xiapex @)}

Le Xiapex est composé de deux collagénases produites par Clostridium histolyticum qui hydrolysent de manière complémentaire le collagène présent au niveau de la plaque de Lapeyronie aboutissant à une digestion de ce collagène après injection [81].

La collagénase de Clostridium histolyticum (Xiapex ${ }^{\circledR}$ ) est le seul traitement doté d'une autorisation de mise sur le marché (AMM) pour la maladie de Lapeyronie.

Deux études de phase III (IMPRESS) comparant la collagenase de Clostridium histolyticum à un placebo sur un total de 832 patients avec des courbures stabilisées entre 30 et 90 degrés recevant au maximum 4 cycles (soit 8 injections) de traitement a mis en évidence une différence significative $(\mathrm{p}<0.0001)$ de réduction de courbure moyenne, à savoir 17 degrés $( \pm 14.8)$ dans le groupe traité versus 9,3 degrés $( \pm 13.6)$ dans le groupe placebo $[81,82]$

L'utilisation du Xiapex est indiquée dans les courbures dorsales stabilisées inférieures à $90^{\circ}$.

L'utilisation de Xiapex est contre-indiquée :

- en cas d'antécédent d'hypersensibilité au Xiapex ou à une autre collagénase utilisée dans le cadre d'une autre indication thérapeutique

- pour le traitement de plaques de Lapeyronie affectant l'urètre (plaque ventrale)

L'injection de Xiapex expose principalement à des risques de rupture des corps caverneux $(0,5$ - 0,9\%), d'ecchymose ou hématome pénien majorés par la prise d'antiagrégants plaquettaires (autorisée jusqu'à $150 \mathrm{mg}$ d'aspirine uniquement).

Le Xiapex était le seul traitement médical à posséder une AMM en traitement de la maladie de Lapeyronie, avec des résultats modérés mais significatifs. Il n'est plus commercialisé depuis le

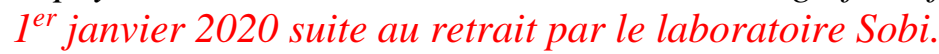

\section{Vérapamil}


Plusieurs études observationnelles ont montré l'efficacité du vérapamil (inhibiteur calcifique) injecté au sein de la plaque dans la stabilisation ou la réduction de la courbure [8386]. Deux études en double aveugle contre placebo n'ont pas démontré de différence significative entre le vérapamil intraplaque et le placebo $[87,88]$.Des recommandations internationales, canadiennes et américaines considèrent l'utilisation du vérapamil en injection intraplaque comme une option de seconde ligne, contrairement aux sociétés savantes européennes qui n'émettent pas de recommandation concernant cette molécule.

Le vérapamil peut être proposé en raison de l'absence du Xiapex, sans niveau de preuve important quant à son efficacité (avis d'expert). Il n'existe pas de consensus quant au dosage et à la fréquence d'administration de cette molécule. Le protocole le plus couramment utilisé consiste en l'injection de $6 \mathrm{ml}$ de vérapamil intraplaque toutes les 3 semaines pouvant être répété 6 fois.

\section{Interféron $\alpha 2 b$}

L'interféron appartient à la famille des cytokines. L'action de l'IFNa2b sur l'inhibition de la production de collagène a engendré des recherches dans la maladie de Lapeyronie [89]. Parmi ces études, l'une d'entre elle a comparé l'IFN $\alpha 2 b$ injecté dans la plaque versus placebo chez plus de 100 patients en double aveugle et a montré une amélioration significative de la courbure du pénis $\left(-13,5^{\circ}\right)$ dans le groupe traité par IFNa2b [90].

Les potentiels effets secondaires de l'IFN $\alpha 2 b$ (myalgies, fièvre et syndrome grippal) peuvent être prévenus par un traitement symptomatique avant l'injection.

L'IFNa2b intra lésionnel n'a pas l'AMM dans la maladie de Lapeyronie mais les résultats positifs des essais, positionne favorablement ce traitement dans les recommandations internationales. Ce traitement reste cependant difficilement accessible en utilisation courante contrairement au vérapamil.

\section{Corticoüdes}

Les injections de corticoïdes ont été réalisées durant de nombreuses années et en particulier au cours de la phase active initiale.

En effet, une étude non randomisée à faible effectif avait mis en évidence une diminution de la taille des plaques et de la douleur [91].

Enfin, une étude en double aveugle contre placebo avec administration intralésionnelle de bétaméthasone n'a retrouvé aucune amélioration statistiquement significative de la déformation du pénis, de la taille de la plaque et de la douleur [92].

Dans l'état actuel des connaissances, il n'existe pas d'argument scientifique pour recommander l'utilisation d'une injection intra-plaque de corticoïde, dans le but d'une réduction de la courbure, ou de la douleur.

\section{CONCLUSION DES TRAITEMENTS PAR INJECTION :}

Aucun produit disponible n'a l'Autorisation de Mise sur le Marché. En l'absence du Xiapex, le vérapamil pourrait être proposé (avis d'expert). Malgré ces traitements, l'évolution naturelle de la maladie peut se poursuivre et n'est pas imputable aux injections intra-plaque. 


\section{THERAPIE PAR TRACTION}

La thérapie par traction est un traitement provoquant un étirement du pénis. Ces dispositifs vont étirer la cicatrice inextensible (plaque) engendrée par maladie de Lapeyronie. Cette contrainte biomécanique va aboutir à un remodelage de la matrice extracellulaire et notamment du collagène via des voies de transduction de signal et des mécanismes de régulation génique [93-95].

Différents dispositifs existent : les extenseurs péniens, le vacuum et les nouveaux dispositifs. Ces thérapeutiques ont l'avantage de ne pas présenter de risque systémique pour les patients, mais restent contraignantes au quotidien. La motivation et l'observance du patient sont donc indispensables pour escompter un effet de ces dispositifs.

Tous ces dispositifs sont non remboursés.

\section{Extenseurs péniens :}

La principale série contrôlée a été réalisée chez 96 patients; dont 55 d'entre eux exerçaient une traction continue 6 à $9 \mathrm{~h}$ par jour pendant 6 à 9 mois VS 41 patients (ayant une thérapie orale uniquement). Une amélioration moyenne significative de la courbure de $20^{\circ} \mathrm{a}$ été observée dans le groupe traité par rapport aux témoins $(\mathrm{p}<0,05)$. La longueur du pénis était également améliorée : $+1,5 \mathrm{~cm}$ contre $-2,6 \mathrm{~cm}(\mathrm{p}=0,03)$, ainsi que sur la douleur et la fonction sexuelle. L'indication chirurgicale a été réduite chez $40 \%$ des patients et a simplifié la procédure chez 1 patient sur 3 . Une utilisation $>6 \mathrm{~h}$ par jour semblait être un facteur positif chez les patients en phase aigüe (28).

Pour être efficace, ce dispositif doit être porté de façon prolongée ( 2 à 8 heures par jour) pendant au moins 6 mois [97,98].

\section{Vacuum :}

Le vacuum est une pompe à dépression active, qui va provoquer un étirement en longueur et en largeur du pénis. La principale étude l'a étudié chez 31 patients atteints de ML. Une diminution de la courbure du pénis de $68 \%$ avec une amélioration de 5 à $25^{\circ}(\mathrm{P}<0,001)$ a été notée après une utilisation de $10 \mathrm{~min}$ deux fois par jour pendant 12 semaines. Une amélioration de la taille du pénis et une diminution de la douleur ont également été retrouvées. Les auteurs suggèrent que le traitement par vacuum pourrait stabiliser une plaque souple [99]. Les thérapies par traction ont également été étudiées dans le cadre de traitement multimodal (cf. paragraphe traitements multimodaux).

\section{Les nouveaux dispositifs :}

Une des premières études [103] a montré une efficacité sur la diminution de la douleur par un traitement par onde de choc, la lithotritie extracorporelle, dans la ML sans efficacité sur la courbure. Il était suggéré une lésion des récepteurs sensoriels à l'origine de cette amélioration. Mais ces ondes de chocs étaient produites par des sondes de haute fréquence, adaptées au traitement des lithiases urinaires. Avec l'apparition des sondes de faibles intensités, utilisées initialement dans le cadre d'une stimulation de la néoangiogénèse chez les patients diabétiques 
présentant des retards de cicatrisation, son utilisation s'est élargie à d'autres indications, notamment dans la dysfonction érectile [104].

ISSM (2016) L'utilisation d'une thérapie par traction peut avoir un bénéfice limité dans la ML AUA (2015) Pas de recommandation

CAU (2018) Recommandation basée sur faible niveau de preuve

EAU (2019) Il est recommandé d'utiliser une thérapie par traction dans le but de réduire la déformation pénienne et de lutter contre le raccourcissement

Au vu de ces résultats, et de l'absence d'effet systémique une thérapie par traction, Il peut être proposé aux patients à la phase active ou stabilisée de la maladie, éventuellement dans le cadre d'un traitement multimodal ou d'une rééducation post opératoire d'une ML.

\section{LES ONDES DE CHOC DE FAIBLE INTENSITE}

Une des premières études [103] a montré une efficacité sur la diminution de la douleur par un traitement par onde de choc, la lithotritie extracorporelle, dans la ML sans efficacité sur la courbure. Il était suggéré une lésion des récepteurs sensoriels à l'origine de cette amélioration. Mais ces ondes de chocs étaient produites par des sondes de haute fréquence, adaptées au traitement des lithiases urinaires. Avec l'apparition des sondes de faibles intensités, utilisées initialement dans le cadre d'une stimulation de la néoangiogénèse chez les patients diabétiques présentant des retards de cicatrisation, son utilisation s'est élargie à d'autres indications, notamment dans la dysfonction érectile [104]. Il existe de nombreuses études observationnelles, 4 études randomisées contrôlées et une méta analyse, réalisées à différentes phases de la maladie. La première étude contrôlée a été réalisée en 2009[100] compara 2 cohortes de 50 patients (ondes VS placebo), avec une évolution inférieure à 12 mois sans traitement préalable, montrant une amélioration de la douleur et de la qualité de vie dans le groupe traité[100]. La seconde randomise 102 patients en simple aveugle, en phase chronique et ayant bénéficié d'un autre traitement au préalable. Celle-ci ne retrouve qu'un effet sur la douleur, avec une tendance à l'aggravation de la courbure dans le groupe traité [101]. La seule méta-analyse ne retient qu'un effet sur la douleur, et une procédure peu risquée[102].

Des recommandations de l'ESSM ont été éditées en 2019, ainsi que par l'EAU, la CAU et l'AUA et considère l'utilisation des ondes de choc possible contre la douleur, sans effet sur la taille de la plaque ni sur la courbure [103].

ISSM (2016) Impact minimal sur la déformation mais produit une diminution rapide de la douleur et la stabilisation de la maladie

AUA (2015) Les ondes de chocs ne doivent pas être utilisées pour réduire la courbure ou la taille de la plaque

CAU (2018) Non recommandé pour réduire la taille de la plaque ou la courbure

EAU (2019) Les ondes de chocs ne permettent pas une réduction de la taille de la plaque ou de la courbure mais peuvent être proposées à visée antalgique

Les ondes de chocs de faible intensité restent un traitement débattu dans la ML. Il n'existe pas de consensus sur le niveau ni le type d'énergie idéal. L'impact rapide sur la douleur est retrouvé dans la plupart des études. Ce traitement peut donc être proposé à visée antalgique dans le cadre de la ML. Un PHRC est en cours afin d'étudier l'effet des ondes de chocs dans la ML. 


\section{LES TRAITEMENTS COMBINES}

Il est possible que le plus grand bénéfice à retenir des traitements mini invasifs (non chirurgicaux) de la ML réside en une utilisation multimodale combinant les thérapies orales, par injection ou par traction. Malgré les effets limités de ces traitements, leur synergie d'action sur les différents mécanismes physiopathologiques de la ML pourrait permettre de limiter la déformation et notamment à la phase active de la maladie [104].

Différents protocoles ont été utilisés : 1'association L-arginine orale, pentoxifylline orale et d'injection intra-plaque de verapamil, avec ou sans extenseur pénien a été testé chez 39 hommes, $54 \%$ avaient une réduction de la courbe $\geq 10^{\circ}$, avec une amélioration moyenne de $26,9^{\circ}$ dans le groupe ayant utilisé la thérapie par traction [105]. L'association d'IPDE 5 avec les injections intra-plaque de collagénase optimisait la correction de la courbure [76]. D'autres études associant les thérapies par traction et les injections intra-plaque (interféron alpha ou collagénase) permettent d'envisager l'utilisation de ces traitements combiné [106,107]. L'association de traitement du type onde de choc avec le tadalafil [75] ou injection de fraction stromale vasculaire [108] ont également été testées, avec des résultats prometteurs dans le cadre des cellules souches, mais restant pour l'instant du domaine de la recherche.

Après une information éclairée sur les bénéfices et les risques attendus, à la phase active de la maladie ou chez des patients refusant les traitements chirurgicaux, un traitement multimodal associant un traitement oral type IPDE5, ou injection intra-plaque associée à une thérapie par traction peut être proposé [80].

\section{NOUVELLES THERAPIES}

De nouveaux traitements ont fait leur apparition ces dernières années, comme les injections de plasma enrichi en plaquettes, d'acide hyaluronique, de cellules souches, ainsi que l'administration de mycophenate mofetil, ou encore l'application de topique H100 [109].

Certains de ces traitements ont déjà étudié chez l'humain dans des cohortes cliniques [110-114]. Bien que la plupart ont été utilisés à la phase stabilisée, certains ciblent la phase active de la maladie [115]. Même si la perspective d'intégrer ces traitements dans la prise en charge de la ML est séduisante, le niveau de preuve de ces études est faible avec des effectifs limités et surtout une absence de comparaisons aux traitements standards.

L'intérêt de ces traitements doit donc être confirmé et ils ne peuvent actuellement pas faire l'objet de recommandations. 
Tableau 2 : récapitulatif des traitements non chirurgicaux

\begin{tabular}{|c|c|c|c|c|c|}
\hline Traitement & $\begin{array}{c}\text { Mode } \\
\text { d'administ } \\
\text { ration }\end{array}$ & Effet & $\begin{array}{l}\text { Utilisation } \\
\text { en } \\
\text { monothéra } \\
\text { pie }\end{array}$ & \begin{tabular}{|c|} 
Utilisation \\
en \\
traitement \\
multimoda \\
I
\end{tabular} & $\begin{array}{l}\text { NIVEAU de } \\
\text { preuve }\end{array}$ \\
\hline IPDE 5 & Orale & $\begin{array}{c}\text { Améliore la } \\
\text { Fonction } \\
\text { érectile }\end{array}$ & \begin{tabular}{|c} 
Oui \\
en cas de \\
DE
\end{tabular} & Oui & Grade A \\
\hline AINS & Orale & $\begin{array}{l}\text { Diminue } \\
\text { les } \\
\text { douleurs }\end{array}$ & \multicolumn{2}{|c|}{$\begin{array}{l}\text { Oui } \\
\text { en cas de douleur }\end{array}$} & $\begin{array}{c}\text { Avis } \\
\text { d'expert }\end{array}$ \\
\hline Vacuum & $\begin{array}{c}\text { Traction } \\
\text { mécanique }\end{array}$ & $\begin{array}{c}\text { Réduis la } \\
\text { déformatio } \\
n ? \\
\text { Améliore la } \\
\text { largeur? }\end{array}$ & \multicolumn{2}{|c|}{$\begin{array}{c}\text { Oui } \\
\text { (20 min par jour en } \\
\text { deux fois) }\end{array}$} & Grade B \\
\hline $\begin{array}{c}\text { Extenseur } \\
\text { pénien }\end{array}$ & $\begin{array}{c}\text { Traction } \\
\text { mécanique }\end{array}$ & \begin{tabular}{|c|} 
Réduis la \\
déformatio \\
$n$ \\
Améliore la \\
taille du \\
pénis
\end{tabular} & \multicolumn{2}{|c|}{$\begin{array}{c}\text { Oui } \\
\text { (> 8h par jour) }\end{array}$} & Grade B \\
\hline \begin{tabular}{|c|} 
Collagénos \\
e de \\
clostridium \\
histolyticu \\
m
\end{tabular} & $\begin{array}{c}\text { Injection } \\
\text { intraplaque }\end{array}$ & $\begin{array}{c}\text { Réduis la } \\
\text { déformatio } \\
n\end{array}$ & \multicolumn{2}{|c|}{$\begin{array}{l}\text { Oui } \\
\text { plus disponible en } \\
\text { France }\end{array}$} & Grade A \\
\hline Verapamil & $\begin{array}{c}\text { Injection } \\
\text { intraplaque }\end{array}$ & $\begin{array}{c}\text { Diminue la } \\
\text { plaque ? }\end{array}$ & Non & \begin{tabular}{|c|} 
Oui \\
Jusqu'à 2 \\
injections \\
par \\
semaines \\
pendant 12 \\
semaines \\
\end{tabular} & $\begin{array}{c}\text { Avis } \\
\text { d'expert }\end{array}$ \\
\hline $\begin{array}{l}\text { Onde de } \\
\text { choc }\end{array}$ & Mécanique & 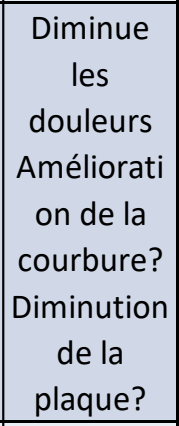 & $\begin{array}{r}\text { Oui, en cas } \\
\text { (1 séance } p \\
\text { pendar } \\
\text { sema }\end{array}$ & $\begin{array}{l}\text { de douleur } \\
\text { oar semaine } \\
\text { nt } 4 \text { à } 6 \\
\text { aines) }\end{array}$ & Grade B \\
\hline $\begin{array}{c}\text { Le } \\
\text { Traitement } \\
\text { multimodal }\end{array}$ & \begin{tabular}{|c|} 
Orale + \\
traction \\
mécanique \\
+ injection \\
intra \\
plaque
\end{tabular} & $\begin{array}{c}\text { Réduis la } \\
\text { déformatio } \\
n\end{array}$ & $\begin{array}{r}\text { Peut-être p } \\
\text { des patient } \\
\text { ne souha } \\
\text { d'opé }\end{array}$ & $\begin{array}{l}\text { rescrit chez } \\
\text { ts motivé ou } \\
\text { aitant pas } \\
\text { gration }\end{array}$ & Grade C \\
\hline
\end{tabular}




\section{TRAITEMENTS CHIRURGICAUX}

\section{Indication}

Pour proposer un traitement chirurgical, la maladie de Lapeyronie doit être :

- Stabilisée : symptômes ayant commencé il y a au moins 6 mois et en général plus

d'un an, avec une stabilité de la déformation et une absence de douleur évolutive depuis plus de 3 à 6 mois

- Responsable d'un handicap sexuel important et d'une insatisfaction sexuelle, en général une déformation significative rendant la pénétration difficile (courbure du pénis $>30^{\circ}$ ) et/ou une dysfonction érectile non répondeuse

Après échec d'un traitement conservateur ou si le patient souhaite un traitement chirurgical. $[47,121]$

\section{Informations et Objectifs}

Avant tout traitement chirurgical, une information claire et objective sur l'efficacité et les complications de l'intervention doit être délivrée au patient. Ses attentes doivent être énoncées et des objectifs réalistes définis. La satisfaction post-opératoire est conditionnée par la compréhension des résultats attendus et de ses limites.

L'objectif principal est d'améliorer les difficultés de pénétration secondaire à la courbure, en conservant une érection rigide et une taille de pénis suffisante.

L'information délivrée insiste sur la diminution de la taille de la verge induite par la maladie de Lapeyronie elle-même, pouvant être accentuée après chirurgie, sur le risque de persistance de la courbure et de sa récidive, d'apparition d'une dysfonction érectile (DE) ou d'aggravation d'une DE préexistante, de trouble de la sensibilité de la verge, de trouble éjaculatoire ou orgasmique, de douleurs, d'hématome et de troubles de la vascularisation, d'infection, de phimosis, de rétention d'urine, de plaie de l'urètre ainsi que des risques spécifiques lors de l'utilisation de matériel prothétique. [117]

Trois stratégies chirurgicales en cas de maladie de Lapeyronie peuvent être proposées :

1. les plicatures

2. les incisions/excisions avec greffon

3. les implants péniens.

\section{Les plicatures - traitement de la convexité de la déformation}

Il s'agit du traitement chirurgical le plus fréquemment pratiqué pour la correction de la courbure. L'objectif est de corriger la courbure en traitant le côté opposé à la plaque responsable de la déformation.

Indications 
Ses indications sont une courbure simple (sans déformation angulaire : encoche en verre de montre, étranglement en sablier...) le plus souvent inférieure à $60^{\circ}$, avec une fonction érectile conservée (avec ou sans traitement pharmacologique ou mécanique) et une taille de verge suffisante.

Pour cette technique une longueur de pénis $>13 \mathrm{~cm}$ en érection a été proposé. ${ }^{(6-7)}$ Cependant des difficultés liées à la longueur lors des rapports sexuels ne sont mentionnées que pour des verges en érections $<10 \mathrm{~cm}^{(8)}$. La notion de raccourcissement prévu de moins de $20 \%$ de la longueur est également utilisée [120]. Actuellement, il n'y a pas de consensus sur la taille minimum, cela doit être discuté avec le patient en prenant en compte la taille et l'élasticité du pénis, le degré et la forme de courbure et les possibilités thérapeutiques.

\section{Techniques}

L'incision cutané est le plus souvent coronale éventuellement associée à une posthectomie, parfois élective selon le type de déformation, le type de plicature et les habitudes du chirurgien.

Plusieurs techniques de plicature ont été décrites, mais aucune n'a fait preuve de sa supériorité pour améliorer l'efficacité et diminuer les effets secondaires [121-126].

Il existe 2 grands types d'opérations, avec ou sans ouverture de l'albuginée des corps caverneux (CC).

Les plicatures avec ouverture de l'albuginée des corps caverneux est une technique initialement effectuée par Nesbit pour les courbures congénitales[121] puis utilisée pour la maladie de Lapeyronie [122] ; Elle consiste à exciser une ellipse d'albuginée aux points de plus grande courbure en face de la position de la plaque et refermer en le suturant horizontalement. Plusieurs modifications de cette technique ont été proposées en réalisant notamment une excision limitée (Diamond shape) ou une incision longitudinale de l'albuginée (Yachia) unie ou multifocale avec suture transversale [128-129].

Pour éviter le traumatisme potentiel lié à l'ouverture des CC, il a été proposé des techniques de de plicatures simples sans ouverture de l'albuginée des corps caverneux (Essed Schroeder, 16 ou 24 dots, Ebbehøj...)[129,130].

\section{Résultats}

La rectitude post opératoire (courbure résiduelle inférieure à $20^{\circ}$ ) est retrouvée en cas de plicatures avec incision de l'albuginée (Nesbit, Yacchia, Diamond shape) dans 42,1\% à $100 \%$ des cas pour des déviations initiales comprises entre $45,5^{\circ}$ à $74,7^{\circ}[123,128,130-132]$.Les principales séries l'estiment respectivement à $89 \%, 86,3 \%$ et $94,6 \%$ [125][126]

Après plicature simple (Essed-Shroeder, 16/24 dots, ...), une verge droite est obtenue chez $57 \%$ à $96 \%$ des patients pour des courbures initiales comprises entre $39^{\circ}$ et $60^{\circ}$. Pour cette technique, les 3 principales publications l'évaluent à $92 \%, 96 \%, 66,2 \%$. [132-139] 
La satisfaction post opératoire varie de 43 à 98,5\% [128,133]. Néanmoins l'absence de standardisation de cette mesure rend l'interprétation difficile, Paez et al considèrent l'insatisfaction comme fréquente chez les patients opérés d'une maladie de Lapeyronie

Après tout type de plicature $47,7 \%$ à $94 \%$ des patients ont des rapports sexuels satisfaisants [129, 130,133-139] et $66 \%$ des partenaires sont satisfaits par l'intervention[129]. La récidive de la déformation varie de (2,1\% et 22,2\%)[132,135-138].

\section{Conséquences et Complications}

En cas de plicature, le raccourcissement du pénis a lieu du côté opposé à la plaque. Son importance dépendant du degré et de la localisation de la courbure. [130] Il est mesuré entre $0,36 \mathrm{~cm}$ et $1.8 \mathrm{~cm}$. [131,135-137] mais la réduction est parfois sévère (> $3 \mathrm{~cm})$. [131]

Dans les 2 premières années suivant la plicature, l'apparition d'une DE ou l'aggravation d'une DE préexistante est faible le plus souvent [139-145], mais peut varier et s'aggraver avec le temps notamment avec l'apparition de facteurs de vieillissement physiologiques et l'apparition de facteurs de comorbidité, rendant l'interprétation des résultats difficiles.[123-136]

Les nœuds sont fréquemment palpables après plicature $(33,3 \%$ à $81 \%)$, mais peu souvent responsables d'une gêne $(1,7 \%$ à $6 \%)$. Pour cette raison, les points peuvent être inversant et utiliser différents types de sutures résorbables et non résorbables. [137-141]

D'autres complications plus rares ont été rapportées avec des douleurs résiduelles : des troubles de sensibilité du gland [134], les plaies de l'urètre [130,136], les rétentions aiguës d'urine, les hématomes péniens, les infections du site opératoire. Le risque de phimosis secondaire est faible et la posthectomie est à proposer au cas par cas avec le patient. [123, 128, 130,134,139-145]

\section{Recommandation : (Niveau de preuve 2,3 ; grade de recommandation $B-C$ )}

Les chirurgies de la convexité sont utilisables en première intention chez des patients présentant une maladie de Lapeyronie stabilisée avec une déformation simple, $<60^{\circ}$ et une taille de pénis suffisante. Les résultats sont satisfaisants pour le traitement de la courbure avec un risque faible de complication. Différentes techniques peuvent être réalisées, mais aucune n'a fait preuve de sa supériorité (l'utilisation de questionnaire adapté et la création d'une base de données permettraient de compare les résultats de ces techniques).

\section{Techniques d'incision avec greffon}

Ces techniques chirurgicales ont pour but de corriger la courbure par intervention directe au niveau de la plaque (concavité). Cette option limite la perte de longueur liée à la chirurgie. Ces techniques sont réservées aux déformations importantes $\left(>60^{\circ}\right)$, lorsque la longueur du pénis parait insuffisante, en cas d'étranglement en sablier ou « verre de montre », chez des patients sans dysfonction érectile ou répondant aux traitements pharmacologiques $[2,4,45,63,116]$.

Pour ces techniques une longueur de pénis $<13 \mathrm{~cm}$ en érection a été proposé. [118,119] Cependant des difficultés liées à la longueur lors des rapports sexuels ne sont mentionnées que 
pour des verges en érections $<10 \mathrm{~cm}$ [136]. Actuellement, il n'y a pas de consensus sur la taille, cela doit être discuté avec le patient en prenant en compte la taille et l'élasticité du pénis, le degré et la forme de courbure et les possibilités thérapeutiques.

Son principe vise à inciser la plaque fibreuse en son point d'inflexion maximal, suivi d'une incision longitudinale en $\mathrm{H}$ ou double $\mathrm{Y}$ inversé, le plus souvent après dissection des bandelettes vasculonerveuses en raison de la fréquence d'une atteinte dorsale de l'albuginée. Dans certains cas, une excision partielle voire totale de la plaque (notamment calcifiée) peut être nécessaire. Le défect albuginéal créé est mesuré en traction maximale et recouvert d'un matériau auto ou hétérologue, en général de taille légèrement supérieur en raison d'une rétraction post-opératoire.

Son risque principal est l'aggravation de la fonction érectile, d'environ $25 \%$ (de 22,5 à $60 \%$ après 5 ans de suivi) [136-139].

De plus, un raccourcissement du pénis peut également apparaitre dans $30 \%$ des cas en post opératoire [140-142].

Une réhabilitation érectile post opératoire précoce est le plus souvent proposée afin d'éviter la rétraction du greffon et donc diminuer la fibrose post opératoire par l'utilisation d'un vacuum ou d'un extenseur pénien et/ou IPDE5 et/ou par un protocole d'injection intra caverneuse, pendant une durée prolongée pouvant aller jusqu'à 6 mois [143].

Ces inconvénients soulignent l'importance de la sélection et de l'information de ces patients.

La première incision-greffe date de 1974, en utilisant du derme autologue [144]. Depuis lors, de nombreux greffons ont été utilisés. Il n'existe pas de greffon idéal. Il est toutefois peu conseillé d'utiliser des greffons non synthétiques. Le choix du greffon doit pouvoir répondre à certains critères :

-bonne tolérance immunitaire et lutte contre l'infection

-réaction inflammatoire minimale

-faible taux de rétraction

-résorption lente

L'avenir de la greffe d'albuginée est probablement issu de l'ingénierie tissulaire, mais à l'heure actuelle ils ne sont pas évalués et disponibles. [63]

\section{Greffons autologues :}

greffe veineuse : après prélèvement de veine saphène ou dorsale de la verge,

avec un taux de récidive de la courbure de $20 \%$, de raccourcissement de $17 \%$. [145,146] greffe de derme : ne peut être recommandé en raison du fort taux d'échec, avec un taux de récidive de $35 \%, 40 \%$ de raccourcissement d'apparition progressive, et un taux de réintervention de l'ordre de $17 \%$. [63,116,137,147]

greffe de tunique vaginale: permettrais une diminution du risque de raccourcissement en raison d'une bonne tolérance d'implantation, mais il n'existe pas d'étude suffisamment robuste pour proposer une recommandation. [148]

greffe de muqueuse buccale: sa simplicité de prélèvement, son potentiel d'élasticité, sa revascularisation rapide fait de la muqueuse buccale un tissu adapté à la reconstruction albuginéale. Les résultats sont encourageant devant l'absence de récidive de la courbure, le retour précoce des érections, mais les études restent de petite taille pour en faire le matériau idéal dans la maladie de Lapeyronie. [63,149] 
péricarde cadavérique (Tutoplast ${ }^{\circledR}$, Coloplast ${ }^{\mathrm{TM}}$ ): permet une bonne fonctionnalité mécanique, avec une bonne satisfaction des patients malgré un taux de récidive de la courbure de 44\% [147], mais reste non disponible en France.

sous muqueuse intestinale de porc (SIS $\left.{ }^{2}, \operatorname{Cook}^{\mathrm{TM}}\right)$ : offre une bonne qualité de reconstruction, avec un bon résultat fonctionnel dans peu d'étude (knoll), et des résultats contradictoires en fonction du nombre de couche du produit, l'utilisation d'un tissu 4 couches semblait offrir de meilleurs résultats à moyen terme que le monocouche. [150,151]

Tachosil ${ }^{\circledR}\left(\right.$ Takeda $\left.^{\mathrm{TM}}\right)$ : cette éponge hémostatique issue de tendon de cheval, et recouverte de fibrinogène et de thrombine une diminution du temps opératoire et une bonne qualité d'hémostase directement sur le tissu caverneux, cependant le suivi très rapporté dans les études ne permet pas d'évaluer l'efficacité de ce tissu à l'heure actuelle $[152,153]$.

L'incision de l'albuginée, le plus souvent suivie de greffe offre de bons résultats chez les hommes présentant une courbure de plus de $60^{\circ}$ ou un étranglement en verre de montre avec une fonction érectile préservée. L'existence d'une courbure ventrale, d'une dysfonction érectile pré-opératoire, l'âge supérieur à 60 ans prédisent un plus fort taux d'échec de cette technique. $[2,3,6,63,154]$

\section{Implant pénien}

En cas de déformation pénienne associée à une dysfonction érectile non répondeuse ou en cas d'intolérance aux traitements pharmacologiques, l'implant pénien est la solution permettant de prendre en charge les 2 troubles simultanément. La correction de la rectitude peut se faire de différentes manières, si l'implantation seule ne suffit pas après un test per-opératoire (courbure résiduelle $>20-30^{\circ}$ ) :

- intervention de Nesbit : avec le risque de raccourcissement lié à la technique (voir procédure de raccourcissement)

- le « modeling » : consiste à imprimer sur le corps caverneux, implant gonflé en son sein, avec les tubulures clampées à l'aide de pinces protégées afin de protéger la pompe, une torsion contraire à la courbure pendant 3 fois 90 secondes. En cas de courbure persistante $>30^{\circ}$, une ouverture de l'albuginée s'impose $[2,3,6,63]$.

- ouverture de l'albuginée : en cas de courbure importante ou résiduelle ou attendue supérieure à $30^{\circ}$, une procédure complémentaire doit être réalisée : [2,3,6]

○ ouverture simple : par une voie coronale le plus souvent et dissection de la bandelette neurovasculaire dorsale avec préservation du fascia de Buck, des incisions multiples sur la plaque de l'albuginée sont effectuées afin de redresser la verge. L'apposition des bandelettes et la fermeture du fascia de Buck suffit à leur fermeture en cas de défect $<2 \mathrm{~cm}[2,6,63]$.

- en cas d'incision avec un défect supérieur à $2 \mathrm{~cm}$ un greffon devra être interposé afin d'éviter les hernies de la prothèse au travers de l'incision du corps caverneux $[2,3,6,63]$.

- dans des cas très sélectionnés, et en cas de raccourcissement de verge majeur du à la MLP entrainant des difficultés attendues de pénétration, une restauration partielle de longueur associée à un implant («sliding » technique) peut être proposée [155]. 
L'utilisation de l'implant pénien dans la maladie de Lapeyronie n'expose pas à plus de risque que dans la population générale, en dehors d'une augmentation du risque de plaie urétrale suite à un implant surgonflé trop longtemps [156]. Le taux de satisfaction de la population générale porteuse d'un implant pénien est évalué à plus de $90 \%$. Toutefois dans le cas de la maladie de Lapeyronie, seulement $79 \%$ des patients s'estiment satisfait de leur implant, essentiellement en raison de la perte de longueur. [157]

\section{Recommandations (Grade B niveau 3)}

Les incisions-greffe sont réservées aux courbures $>60^{\circ}$ avec une fonction érectile conservée (Grade B, niveau 3). Cette procédure peut donner de bons résultats chez une équipe entrainée, un patient jeune avec une érection normale, sans facteurs de risque associés.

Le faible niveau de preuve des études cliniques ne permet pas de dégager un niveau de preuve suffisant pour émettre une recommandation forte. Aucun greffon n'a prouvé sa supériorité, tout comme les différentes techniques proposées.

L'utilisation de l'implant pénien permet de traiter les patients présentant une dysfonction érectile réfractaire associée à une courbure importante (Grade B niveau 3).

L'utilisation de traitement mécanique (vacuum, extenseur pénien) en post opératoire est fortement recommandé afin d'optimiser les résultats à moyen et long terme de cette chirurgie.

\section{Bibliographie :}

[1] Bella AJ, Perelman MA, Brant WO, Lue TF. Peyronie's disease (CME). The Journal of Sexual Medicine 2007;4:1527-38. https://doi.org/10.1111/j.1743-6109.2007.00614.x.

[2] Bella AJ, Lee JC, Grober ED, Carrier S, Benard F, Brock GB. 2018 Canadian Urological Association guideline for Peyronie's disease and congenital penile curvature. Canadian Urological Association Journal 2018;12. https://doi.org/10.5489/cuaj.5255.

[3] Chung E, Ralph D, Kagioglu A, Garaffa G, Shamsodini A, Bivalacqua T, et al. Evidence-Based Management Guidelines on Peyronie's Disease. The Journal of Sexual Medicine 2016;13:905-23. https://doi.org/10.1016/j.jsxm.2016.04.062.

[4] Chung E, Wang R, Ralph D, Levine L, Brock G. A Worldwide Survey on Peyronie's Disease Surgical Practice Patterns Among Surgeons. J Sex Med 2018;15:568-75. https://doi.org/10.1016/j.jsxm.2018.01.025.

[5] Hatzimouratidis K, Eardley I, Giuliano F, Hatzichristou D, Moncada I, Salonia A, et al. EAU guidelines on penile curvature. Eur Urol 2012;62:543-52. https://doi.org/10.1016/j.eururo.2012.05.040.

[6] Nehra A, Alterowitz R, Culkin DJ, Faraday MM, Hakim LS, Heidelbaugh JJ, et al. Peyronie's Disease: AUA Guideline. J Urol 2015;194:745-53.

https://doi.org/10.1016/j.juro.2015.05.098. 
[7] Mulhall JP, Creech SD, Boorjian SA, Ghaly S, Kim ED, Moty A, et al. Subjective and objective analysis of the prevalence of Peyronie's disease in a population of men presenting for prostate cancer screening. The Journal of Urology 2004;171:2350-3.

https://doi.org/10.1097/01.ju.0000127744.18878.f1.

[8] Montorsi F, Adaikan G, Becher E, Giuliano F, Khoury S, Lue TF, et al. Summary of the recommendations on sexual dysfunctions in men. J Sex Med 2010;7:3572-88. https://doi.org/10.1111/j.1743-6109.2010.02062.x.

[9] Tal R, Heck M, Teloken P, Siegrist T, Nelson CJ, Mulhall JP. Peyronie's disease following radical prostatectomy: incidence and predictors. J Sex Med 2010;7:1254-61. https://doi.org/10.1111/j.1743-6109.2009.01655.x.

[10] Grasso M, Lania C, Blanco S, Limonta G. The natural history of Peyronie's disease. Archivos Espanoles De Urologia 2007;60:326-31. https://doi.org/10.4321/s000406142007000300021.

[11] Haag SM, Hauck EW, Szardening-Kirchner C, Diemer T, Cha E-S, Weidner W, et al. Alterations in the transforming growth factor (TGF)-beta pathway as a potential factor in the pathogenesis of Peyronie's disease. European Urology 2007;51:255-61.

https://doi.org/10.1016/j.eururo.2006.05.002.

[12] Mulhall JP. Expanding the paradigm for plaque development in Peyronie's disease. International Journal of Impotence Research 2003;15 Suppl 5:S93-102. https://doi.org/10.1038/sj.ijir.3901082.

[13] Gonzalez-Cadavid NF, Rajfer J. Experimental models of Peyronie's disease. Implications for new therapies. The Journal of Sexual Medicine 2009;6:303-13. https://doi.org/10.1111/j.1743-6109.2008.01104.x.

[14] Mulhall JP, Schiff J, Guhring P. An analysis of the natural history of Peyronie's disease. J Urol 2006;175:2115-8; discussion 2118. https://doi.org/10.1016/S00225347(06)00270-9.

[15] Mulhall JP, Anderson MS, Lubrano T, Shankey TV. Peyronie's disease cell culture models: phenotypic, genotypic and functional analyses. International Journal of Impotence Research 2002;14:397-405. https://doi.org/10.1038/sj.ijir.3900874.

[16] Mulhall JP, Thom J, Lubrano T, Shankey TV. Basic fibroblast growth factor expression in Peyronie's disease. The Journal of Urology 2001;165:419-23.

https://doi.org/10.1097/00005392-200102000-00016.

[17] Davila HH, Magee TR, Zuniga FI, Rajfer J, Gonzalez-Cadavid NF. Peyronie's disease associated with increase in plasminogen activator inhibitor in fibrotic plaque. Urology 2005;65:645-8. https://doi.org/10.1016/j.urology.2005.01.010.

[18] Piao S, Ryu J-K, Shin H-Y, Zhang L, Song SU, Han J-Y, et al. Repeated intratunical injection of adenovirus expressing transforming growth factor-beta1 in a rat induces penile curvature with tunical fibrotic plaque: a useful model for the study of Peyronie's disease. International Journal of Andrology 2008;31:346-53. https://doi.org/10.1111/j.13652605.2007.00780.x.

[19] Gelman J, Garbán H, Shen R, Ng C, Cai L, Rajfer J, et al. Transforming growth factor-beta1 (TGF-beta1) in penile and prostate growth in the rat during sexual maturation. Journal of Andrology 1998;19:50-7.

[20] Lin G, Shindel AW, Banie L, Ning H, Huang YC, Liu G, et al. Pentoxifylline attenuates transforming growth factor-beta1-stimulated elastogenesis in human tunica albuginea-derived fibroblasts part 2: Interference in a TGF-beta1/Smad-dependent mechanism and downregulation of AAT1. J Sex Med 7:1787-97. https://doi.org/10.1111/j.17436109.2010.01749.x.

[21] Shindel AW, Lin G, Ning H, Banie L, Huang YC, Liu G, et al. Pentoxifylline attenuates transforming growth factor-beta1-stimulated collagen deposition and elastogenesis 
in human tunica albuginea-derived fibroblasts part 1: impact on extracellular matrix. J Sex Med 7:2077-85. https://doi.org/10.1111/j.1743-6109.2010.01790.x.

[22] El-Sakka AI, Hassoba HM, Pillarisetty RJ, Dahiya R, Lue TF. Peyronie's disease is associated with an increase in transforming growth factor-beta protein expression. The Journal of Urology 1997;158:1391-4.

[23] Lin CS, Lin G, Wang Z, Maddah SA, Lue TF. Upregulation of monocyte chemoattractant protein 1 and effects of transforming growth factor-beta 1 in Peyronie's disease. Biochemical and Biophysical Research Communications 2002;295:1014-9. https://doi.org/10.1016/s0006-291x(02)00765-9.

[24] Lindsay MB, Schain DM, Grambsch P, Benson RC, Beard CM, Kurland LT. The incidence of Peyronie's disease in Rochester, Minnesota, 1950 through 1984. J Urol 1991;146:1007-9. https://doi.org/10.1016/s0022-5347(17)37988-0.

[25] Sommer F, Schwarzer U, Wassmer G, Bloch W, Braun M, Klotz T, et al. Epidemiology of Peyronie's disease. Int J Impot Res 2002;14:379-83. https://doi.org/10.1038/sj.ijir.3900863.

[26] Kadioglu A, Tefekli A, Erol B, Oktar T, Tunc M, Tellaloglu S. A retrospective review of 307 men with Peyronie's disease. J Urol 2002;168:1075-9.

https://doi.org/10.1097/01.ju.0000024040.55662.36.

[27] Rhoden EL, Riedner CE, Fuchs SC, Fuchs S, Ribeiro EP, Halmenschlager G. A crosssectional study for the analysis of clinical, sexual and laboratory conditions associated to Peyronie's disease. J Sex Med 2010;7:1529-37. https://doi.org/10.1111/j.17436109.2009.01584.x.

[28] Bjekic MD, Vlajinac HD, Sipetic SB, Marinkovic JM. Risk factors for Peyronie's disease: a case-control study. BJU Int 2006;97:570-4. https://doi.org/10.1111/j.1464410X.2006.05969.x.

[29] Ralph D, Gonzalez-Cadavid N, Mirone V, Perovic S, Sohn M, Usta M, et al. The management of Peyronie's disease: evidence-based 2010 guidelines. J Sex Med 7:2359-74. https://doi.org/10.1111/j.1743-6109.2010.01850.x.

[30] Bekos A, Arvaniti M, Hatzimouratidis K, Moysidis K, Tzortzis V, Hatzichristou D. The natural history of Peyronie's disease: an ultrasonography-based study. Eur Urol 2008;53:644-50. https://doi.org/10.1016/j.eururo.2007.07.013.

[31] Pryor J, Akkus E, Alter G, Jordan G, Lebret T, Levine L, et al. Peyronie's disease. J Sex Med 2004;1:110-5. https://doi.org/10.1111/j.1743-6109.2004.10116.x.

[32] Mulhall JP, Goldstein I, Bushmakin AG, Cappelleri JC, Hvidsten K. Validation of the erection hardness score. J Sex Med 2007;4:1626-34. https://doi.org/10.1111/j.1743-

6109.2007.00600.x.

[33] DeLay KJ, Haney N, Hellstrom WJ. Modifying Risk Factors in the Management of Erectile Dysfunction: A Review. World J Mens Health 2016;34:89-100. https://doi.org/10.5534/wjmh.2016.34.2.89.

[34] Coyne KS, Currie BM, Thompson CL, Smith TM. The test-retest reliability of the Peyronie's disease questionnaire. J Sex Med 2015;12:543-8.

https://doi.org/10.1111/jsm.12769.

[35] Rosen R, Catania J, Lue T, Althof S, Henne J, Hellstrom W, et al. Impact of Peyronie's disease on sexual and psychosocial functioning: qualitative findings in patients and controls. J Sex Med 2008;5:1977-84. https://doi.org/10.1111/j.1743-6109.2008.00883.x. [36] Terrier JE, Nelson CJ. Psychological aspects of Peyronie's disease. Transl Androl Urol 2016;5:290-5. https://doi.org/10.21037/tau.2016.05.14.

[37] Davis SNP, Ferrar S, Sadikaj G, Gerard M, Binik YM, Carrier S. Female Partners of Men With Peyronie's Disease Have Impaired Sexual Function, Satisfaction, and Mood, While 
Degree of Sexual Interference Is Associated With Worse Outcomes. J Sex Med 2016;13:1095-103. https://doi.org/10.1016/j.jsxm.2016.04.074.

[38] Levine LA, Greenfield JM. Establishing a standardized evaluation of the man with Peyronie's disease. Int J Impot Res 2003;15 Suppl 5:S103-112.

https://doi.org/10.1038/sj.ijir.3901083.

[39] Ohebshalom M, Mulhall J, Guhring P, Parker M. Measurement of penile curvature in Peyronie's disease patients: comparison of three methods. J Sex Med 2007;4:199-203. https://doi.org/10.1111/j.1743-6109.2006.00404.x.

[40] Hellstrom WJG, Feldman R, Rosen RC, Smith T, Kaufman G, Tursi J. Bother and distress associated with Peyronie's disease: validation of the Peyronie's disease questionnaire. J Urol 2013;190:627-34. https://doi.org/10.1016/j.juro.2013.01.090.

[41] Kadioğlu A, Tefekli A, Erol H, Cayan S, Kandirali E. Color Doppler ultrasound assessment of penile vascular system in men with Peyronie's disease. Int J Impot Res 2000;12:263-7. https://doi.org/10.1038/sj.ijir.3900569.

[42] Chung E, Yan H, De Young L, Brock GB. Penile Doppler sonographic and clinical characteristics in Peyronie's disease and/or erectile dysfunction: an analysis of 1500 men with male sexual dysfunction. BJU Int 2012;110:1201-5. https://doi.org/10.1111/j.1464410X.2011.10851.x.

[43] McMahon CG. Current diagnosis and management of erectile dysfunction. Med J Aust 2019;210:469-76. https://doi.org/10.5694/mja2.50167.

[44] Sullivan J, Moskovic D, Nelson C, Levine L, Mulhall J. Peyronie's disease: urologist's knowledge base and practice patterns. Andrology 2015;3:260-4.

https://doi.org/10.1111/andr.292.

[45] Nehra A, Alterowitz R, Culkin DJ, Faraday MM, Hakim LS, Heidelbaugh JJ, et al. Peyronie's Disease: AUA Guideline. The Journal of Urology 2015;194:745-53. https://doi.org/10.1016/j.juro.2015.05.098.

[46] Abdessater M, Kanbar A, Gas J, Bart S, Coloby P, Beley S, et al. [Non-surgical management of Peyronie's disease: State of current knowledge]. Prog Urol 2020. https://doi.org/10.1016/j.purol.2020.03.002.

[47] Weidner W, Hauck EW, Schnitker J. Potassium Paraaminobenzoate (POTABA ${ }^{\mathrm{TM}}$ ) in the Treatment of Peyronie's Disease: A Prospective, Placebo-Controlled, Randomized Study. European Urology 2005;47:530-6. https://doi.org/10.1016/j.eururo.2004.12.022.

[48] Magnusson M, Höglund P, Johansson K, Jönsson C, Killander F, Malmström P, et al. Pentoxifylline and vitamin $\mathrm{E}$ treatment for prevention of radiation-induced side-effects in women with breast cancer: A phase two, double-blind, placebo-controlled randomised clinical trial (Ptx-5). European Journal of Cancer 2009;45:2488-95.

https://doi.org/10.1016/j.ejca.2009.05.015.

[49] Hellstrom WJG. Medical Management of Peyronie's Disease. Journal of Andrology 2009;30:397-405. https://doi.org/10.2164/jandrol.108.006221.

[50] Hauck EW, Diemer T, Schmelz HU, Weidner W. A Critical Analysis of Nonsurgical Treatment of Peyronie's Disease. European Urology 2006;49:987-97.

https://doi.org/10.1016/j.eururo.2006.02.059.

[51] Prieto Castro RM, Leva Vallejo ME, Regueiro Lopez JC, Anglada Curado FJ, Alvarez Kindelan J, Requena Tapia MJ. Combined treatment with vitamin $\mathrm{E}$ and colchicine in the early stages of Peyronie's disease. BJU Int 2003;91:522-4.

[52] Keith ME, Jeejeebhoy KN, Langer A, Kurian R, Barr A, O’Kelly B, et al. A controlled clinical trial of vitamin $\mathrm{E}$ supplementation in patients with congestive heart failure. The American Journal of Clinical Nutrition 2001;73:219-24.

https://doi.org/10.1093/ajen/73.2.219. 
[53] Hashimoto K, Hisasue S-I, Kato R, Kobayashi K, Shimizu T, Tsukamoto T. Outcome analysis for conservative management of Peyronie's disease: Conservative management of Peyronie's disease. International Journal of Urology 2006;13:244-7.

https://doi.org/10.1111/j.1442-2042.2006.01270.x.

[54] Inal T, Tokatli Z, Akand M, Özdiler E, Yaman Ö. Effect of intralesional interferonalpha $2 \mathrm{~b}$ combined with oral vitamin $\mathrm{E}$ for treatment of early stage Peyronie's disease: A randomized and prospective study. Urology 2006;67:1038-42.

https://doi.org/10.1016/j.urology.2005.11.005.

[55] Safarinejad MR, Hosseini SY, Kolahi AA. Comparison of Vitamin E and Propionyl-LCarnitine, Separately or in Combination, in Patients With Early Chronic Peyronie's Disease:

A Double-Blind, Placebo Controlled, Randomized Study. The Journal of Urology 2007;178:1398-403. https://doi.org/10.1016/j.juro.2007.05.162.

[56] Paulis G, Brancato T, D’Ascenzo R, De Giorgio G, Nupieri P, Orsolini G, et al. Efficacy of vitamin $\mathrm{E}$ in the conservative treatment of Peyronie's disease: legend or reality? A controlled study of 70 cases. Andrology 2013;1:120-8. https://doi.org/10.1111/j.20472927.2012.00007.x.

[57] Abner EL, Schmitt FA, Mendiondo MS, Marcum JL, Kryscio RJ. Vitamin E and allcause mortality: a meta-analysis. Curr Aging Sci 2011;4:158-70.

[58] Jordan GH, Carson CC, Lipshultz LI. Minimally invasive treatment of Peyronie's disease: evidence-based progress: Minimally invasive treatment of Peyronie's disease. BJU Int 2014;114:16-24. https://doi.org/10.1111/bju.12634.

[59] Akkus E, Carrier S, Rehman J, Breza J, Kadioglu A, Lue TF. Is colchicine effective in Peyronie's disease? A pilot study. Urology 1994;44:291-5.

[60] Kadioğlu A, Tefekli A, Köksal T, Usta M, Erol H. Treatment of Peyronie's disease with oral colchicine: long-term results and predictive parameters of successful outcome. Int $\mathbf{J}$ Impot Res 2000;12:169-75. https://doi.org/10.1038/sj.ijir.3900519.

[61] Safarinejad MR. Therapeutic effects of colchicine in the management of Peyronie's disease: a randomized double-blind, placebo-controlled study. International Journal of Impotence Research 2004;16:238-43. https://doi.org/10.1038/sj.ijir.3901185.

[62] Cocco G, Chu DCC, Pandolfi S. Colchicine in clinical medicine. A guide for internists. European Journal of Internal Medicine 2010;21:503-8.

https://doi.org/10.1016/j.ejim.2010.09.010.

[63] Hatzimouratidis K, Eardley I, Giuliano F, Hatzichristou D, Moncada I, Salonia A, et al. EAU Guidelines on Penile Curvature. European Urology 2012;62:543-52. https://doi.org/10.1016/j.eururo.2012.05.040.

[64] Ralph DJ, Brooks MD, Bottazzo GF, Pryor JP. The treatment of Peyronie's disease with tamoxifen. Br J Urol 1992;70:648-51.

[65] Teloken C, Rhoden EL, Grazziotin TM, Ros CT, Sogari PR, Souto CA. Tamoxifen versus placebo in the treatment of Peyronie's disease. J Urol 1999;162:2003-5.

[66] Lin G, Shindel AW, Banie L, Ning H, Huang Y-C, Liu G, et al. Pentoxifylline Attenuates Transforming Growth Factor- $\beta 1$-Stimulated Elastogenesis in Human Tunica Albuginea-Derived Fibroblasts Part 2: Interference in a TGF- $\beta 1 /$ Smad-Dependent Mechanism and Downregulation of AAT1. The Journal of Sexual Medicine 2010;7:1787-97. https://doi.org/10.1111/j.1743-6109.2010.01749.x.

[67] Ibrahim A, Gazzard L, Alharbi M, Rompré-Brodeur A, Aube M, Carrier S. Evaluation of Oral Pentoxifylline, Colchicine, and Penile Traction for the Management of Peyronie's Disease. Sex Med 2019;7:459-63. https://doi.org/10.1016/j.esxm.2019.07.003.

[68] Safarinejad MR, Asgari MA, Hosseini SY, Dadkhah F. A double-blind placebocontrolled study of the efficacy and safety of pentoxifylline in early chronic Peyronie's 
disease: PENTOXIFYLLINE IN EARLY PEYRONIE'S DISEASE. BJU International 2009;106:240-8. https://doi.org/10.1111/j.1464-410X.2009.09041.x.

[69] Smith JF, Shindel AW, Huang Y-C, Clavijo RI, Flechner L, Breyer BN, et al. Pentoxifylline treatment and penile calcifications in men with Peyronie's disease. Asian Journal of Andrology 2011;13:322-5. https://doi.org/10.1038/aja.2010.117.

[70] Colson MH, Cuzin B, Faix A, Grellet L, Huyghes E. Les traitements oraux de la dysfonction érectile aujourd'hui, pour quel patient ? Sexologies 2018;27:37-46.

https://doi.org/10.1016/j.sexol.2018.01.009.

[71] Mulhall JP, Schiff J, Guhring P. An analysis of the natural history of Peyronie's disease. J Urol 2006;175:2115-8; discussion 2118. https://doi.org/10.1016/S00225347(06)00270-9.

[72] Burri A, Porst H. The relationship between penile deformity, age, psychological bother, and erectile dysfunction in a sample of men with Peyronie's Disease (PD). Int J Impot Res 2018;30:171-8. https://doi.org/10.1038/s41443-018-0029-3.

[73] Levine LA, Latchamsetty KC. Treatment of erectile dysfunction in patients with Peyronie's disease using sildenafil citrate. Int J Impot Res 2002;14:478-82. https://doi.org/10.1038/sj.ijir.3900912.

[74] Ozturk U, Yesil S, Goktug HNG, Gucuk A, Tuygun C, Sener NC, et al. Effects of sildenafil treatment on patients with Peyronie's disease and erectile dysfunction. Ir J Med Sci 2014;183:449-53. https://doi.org/10.1007/s11845-013-1036-5.

[75] Palmieri A, Imbimbo C, Creta M, Verze P, Fusco F, Mirone V. Tadalafil once daily and extracorporeal shock wave therapy in the management of patients with Peyronie's disease and erectile dysfunction: results from a prospective randomized trial. Int $\mathbf{J}$ Androl

2012;35:190-5. https://doi.org/10.1111/j.1365-2605.2011.01226.x.

[76] Cocci A, Cito G, Urzì D, Minervini A, Di Maida F, Sessa F, et al. Sildenafil $25 \mathrm{mg}$ ODT + Collagenase Clostridium hystoliticum vs Collagenase Clostridium hystoliticum Alone for the Management of Peyronie's Disease: A Matched-Pair Comparison Analysis. J Sex Med 2018;15:1472-7. https://doi.org/10.1016/j.jsxm.2018.08.012.

[77] Gonzalez-Cadavid NF, Rajfer J. Treatment of Peyronie's disease with PDE5 inhibitors: an antifibrotic strategy. Nat Rev Urol 2010;7:215-21.

https://doi.org/10.1038/nrurol.2010.24.

[78] Chung E, Deyoung L, Brock GB. The role of PDE5 inhibitors in penile septal scar remodeling: assessment of clinical and radiological outcomes. J Sex Med 2011;8:1472-7. https://doi.org/10.1111/j.1743-6109.2011.02217.x.

[79] Burnett AL, Nehra A, Breau RH, Culkin DJ, Faraday MM, Hakim LS, et al. Erectile Dysfunction: AUA Guideline. Journal of Urology 2018;200:633-41.

https://doi.org/10.1016/j.juro.2018.05.004.

[80] Farrell MR, Ziegelmann MJ, Levine LA. Minimally invasive therapies for Peyronie's disease: the current state of the art. Translational Andrology and Urology 2020;9:S269-83. https://doi.org/10.21037/tau.2019.08.06.

[81] Lipshultz LI, Goldstein I, Seftel AD, Kaufman GJ, Smith TM, Tursi JP, et al. Clinical efficacy of collagenase Clostridium histolyticum in the treatment of Peyronie's disease by subgroup: results from two large, double-blind, randomized, placebo-controlled, phase III studies. BJU Int 2015;116:650-6. https://doi.org/10.1111/bju.13096.

[82] Gelbard M, Goldstein I, Hellstrom WJG, McMahon CG, Smith T, Tursi J, et al. Clinical efficacy, safety and tolerability of collagenase clostridium histolyticum for the treatment of peyronie disease in 2 large double-blind, randomized, placebo controlled phase 3 studies. J Urol 2013;190:199-207. https://doi.org/10.1016/j.juro.2013.01.087.

[83] Heidari M, Nejadi JR, Ghate A, Delfan B, Iran-Pour E. Evaluation of intralesional injection of verapamil in treatment of Peyronie's disease. J Pak Med Assoc 2010;60:291-3. 
[84] Moskovic DJ, Alex B, Choi JM, Nelson CJ, Mulhall JP. Defining predictors of response to intralesional verapamil injection therapy for Peyronie's disease. BJU Int 2011;108:1485-9. https://doi.org/10.1111/j.1464-410X.2010.10029.x.

[85] Bennett NE, Guhring P, Mulhall JP. Intralesional verapamil prevents the progression of Peyronie's disease. Urology 2007;69:1181-4.

https://doi.org/10.1016/j.urology.2007.02.042.

[86] Wolff B, Peyronnet B, Cattarino S, Mozer P, Renard-Penna R, Phé V, et al.

Intralesional Injections for Early Peyronie Disease: Standardized Assessment and Analysis of Predictive Factors for Treatment Response. Urology 2015;86:57-61.

https://doi.org/10.1016/j.urology.2015.03.010.

[87] Shirazi M, Haghpanah AR, Badiee M, Afrasiabi MA, Haghpanah S. Effect of intralesional verapamil for treatment of Peyronie's disease: a randomized single-blind, placebo-controlled study. Int Urol Nephrol 2009;41:467-71. https://doi.org/10.1007/s11255009-9522-4.

[88] Rehman J, Benet A, Melman A. Use of intralesional verapamil to dissolve Peyronie's disease plaque: a long-term single-blind study. Urology 1998;51:620-6.

https://doi.org/10.1016/s0090-4295(97)00700-0.

[89] Ahuja S, Bivalacqua TJ, Case J, Vincent M, Sikka SC, Hellstrom WJ. A pilot study demonstrating clinical benefit from intralesional interferon alpha $2 \mathrm{~B}$ in the treatment of Peyronie's disease. J Androl 1999;20:444-8.

[90] Hellstrom WJG, Kendirci M, Matern R, Cockerham Y, Myers L, Sikka SC, et al. Single-blind, multicenter, placebo controlled, parallel study to assess the safety and efficacy of intralesional interferon alpha-2B for minimally invasive treatment for Peyronie's disease. J Urol 2006;176:394-8. https://doi.org/10.1016/S0022-5347(06)00517-9.

[91] Winter CC, Khanna R. Peyronie's disease: results with dermo-jet injection of dexamethasone. J Urol 1975;114:898-900.

[92] Cipollone G, Nicolai M, Mastroprimiano G, Iantorno R, Longeri D, Tenaglia R.

[Betamethasone versus placebo in Peyronie's disease]. Arch Ital Urol Androl 1998;70:165-8.

[93] Li J, Wang S, Qin F, Zhu M, You X, Wu C, et al. Reduction in Peyronie's-like plaque size using a vacuum erection device in a rat model of Peyronie's disease via the TGF-

$\beta$ /SMAD signalling pathway. Andrologia 2018;50:e13051. https://doi.org/10.1111/and.13051.

[94] Chung E, De Young L, Solomon M, Brock GB. Peyronie's Disease and

Mechanotransduction: An In Vitro Analysis of the Cellular Changes to Peyronie's Disease in a Cell-Culture Strain System. The Journal of Sexual Medicine 2013;10:1259-67. https://doi.org/10.1111/jsm.12082.

[95] Yuan J, Lin H, Li P, Zhang R, Luo A, Berardinelli F, et al. Molecular Mechanisms of Vacuum Therapy in Penile Rehabilitation: A Novel Animal Study. European Urology 2010;58:773-80. https://doi.org/10.1016/j.eururo.2010.07.005.

[96] Martínez-Salamanca JI, Egui A, Moncada I, Minaya J, Ballesteros CM, del Portillo L, et al. Acute Phase Peyronie's Disease Management with Traction Device: A Nonrandomized Prospective Controlled Trial with Ultrasound Correlation. The Journal of Sexual Medicine 2014;11:506-15. https://doi.org/10.1111/jsm.12400.

[97] Levine LA, Newell M, Taylor FL. Penile Traction Therapy for Treatment of Peyronie's Disease: A Single-Center Pilot Study. The Journal of Sexual Medicine 2008;5:1468-73. https://doi.org/10.1111/j.1743-6109.2008.00814.x.

[98] Gontero P, Di Marco M, Giubilei G, Bartoletti R, Pappagallo G, Tizzani A, et al. ORIGINAL RESEARCH-PEYRONIE'S DISEASE: Use of Penile Extender Device in the Treatment of Penile Curvature as a Result of Peyronie's Disease. Results of a Phase II Prospective Study. The Journal of Sexual Medicine 2009;6:558-66. https://doi.org/10.1111/j.1743-6109.2008.01108.x. 
[99] Raheem AA, Garaffa G, Raheem TA, Dixon M, Kayes A, Christopher N, et al. The role of vacuum pump therapy to mechanically straighten the penis in Peyronie's disease: VACUUM PUMP THERAPY TO STRAIGHTEN THE PENIS IN PEYRONIE'S DISEASE. BJU International 2010;106:1178-80. https://doi.org/10.1111/j.1464-410X.2010.09365.x. [100] Palmieri A, Imbimbo C, Longo N, Fusco F, Verze P, Mangiapia F, et al. A first prospective, randomized, double-blind, placebo-controlled clinical trial evaluating extracorporeal shock wave therapy for the treatment of Peyronie's disease. Eur Urol 2009;56:363-9. https://doi.org/10.1016/j.eururo.2009.05.012.

[101] Lebret T, Loison G, Hervé J-M, Mc Eleny KR, Lugagne P-M, Yonneau L, et al. Extracorporeal shock wave therapy in the treatment of Peyronie's disease: experience with standard lithotriptor (siemens-multiline). Urology 2002;59:657-61. https://doi.org/10.1016/s0090-4295(02)01527-3.

[102] Gao L, Qian S, Tang Z, Li J, Yuan J. A meta-analysis of extracorporeal shock wave therapy for Peyronie's disease. Int J Impot Res 2016;28:161-6. https://doi.org/10.1038/ijir.2016.24.

[103] Capogrosso P, Frey A, Jensen CFS, Rastrelli G, Russo GI, Torremade J, et al. LowIntensity Shock Wave Therapy in Sexual Medicine-Clinical Recommendations from the European Society of Sexual Medicine (ESSM). J Sex Med 2019;16:1490-505. https://doi.org/10.1016/j.jsxm.2019.07.016.

[104] Tsambarlis P, Levine LA. Nonsurgical management of Peyronie's disease n.d.:15. [105] Abern MR, Larsen S, Levine LA. Combination of penile traction, intralesional verapamil, and oral therapies for Peyronie's disease. J Sex Med 2012;9:288-95. https://doi.org/10.1111/j.1743-6109.2011.02519.x.

[106] Ralph DJ, Abdel Raheem A, Liu G. Treatment of Peyronie's Disease With Collagenase Clostridium histolyticum and Vacuum Therapy: A Randomized, Open-Label Pilot Study. J Sex Med 2017;14:1430-7. https://doi.org/10.1016/j.jsxm.2017.08.015. [107] Yafi FA, Pinsky MR, Stewart C, Sangkum P, Ates E, Trost LW, et al. The Effect of Duration of Penile Traction Therapy in Patients Undergoing Intralesional Injection Therapy for Peyronie's Disease. J Urol 2015;194:754-8. https://doi.org/10.1016/j.juro.2015.03.092. [108] Lander EB, Berman MH, See JR. Stromal Vascular Fraction Combined with Shock Wave for the Treatment of Peyronie's Disease. Plast Reconstr Surg Glob Open 2016;4:e631. https://doi.org/10.1097/GOX.0000000000000622.

[109] Aditya I, Kwong JCC, Krakowsky Y, Grober ED. Non-conventional therapies for Peyronie's disease: what is the evidence for efficacy? Translational Andrology and Urology 2020;9:S295-302. https://doi.org/10.21037/tau.2019.09.36.

[110] Zucchi A, Costantini E, Cai T, Cavallini G, Liguori G, Favilla V, et al. Intralesional Injection of Hyaluronic Acid in Patients Affected With Peyronie's Disease: Preliminary Results From a Prospective, Multicenter, Pilot Study. Sex Med 2016;4:e83-88. https://doi.org/10.1016/j.esxm.2016.01.002.

[111] Virag R, Sussman H, Lambion S, de Fourmestraux V. Evaluation of the benefit of using a combination of autologous platelet rich-plasma and hyaluronic acid for the treatment of Peyronie's disease. Sexual Health Issues 2017;1. https://doi.org/10.15761/SHI.1000102. [112] Favilla V, Russo GI, Zucchi A, Siracusa G, Privitera S, Cimino S, et al. Evaluation of intralesional injection of hyaluronic acid compared with verapamil in Peyronie's disease: preliminary results from a prospective, double-blinded, randomized study. Andrology 2017;5:771-5. https://doi.org/10.1111/andr.12368.

[113] Matz EL, Pearlman AM, Terlecki RP. Safety and feasibility of platelet rich fibrin matrix injections for treatment of common urologic conditions. Investig Clin Urol 2018;59:61-5. https://doi.org/10.4111/icu.2018.59.1.61. 
[114] Levy JA, Marchand M, Iorio L, Zribi G, Zahalsky MP. Effects of Stem Cell Treatment in Human Patients With Peyronie Disease. J Am Osteopath Assoc 2015;115:e8-13. https://doi.org/10.7556/jaoa.2015.124.

[115] Twidwell J, Levine L. Topical treatment for acute phase Peyronie's disease utilizing a new gel, H-100: a randomized, prospective, placebo-controlled pilot study. Int J Impot Res 2016;28:41-5. https://doi.org/10.1038/ijir.2015.22.

[116] Chung E, Ralph D, Kagioglu A, Garaffa G, Shamsodini A, Bivalacqua T, et al. Evidence-Based Management Guidelines on Peyronie's Disease. The Journal of Sexual Medicine 2016;13:905-23. https://doi.org/10.1016/j.jsxm.2016.04.062.

[117] Sherer BA, Levine LA. Contemporary Review of Treatment Options for Peyronie's Disease. Urology 2016;95:16-24. https://doi.org/10.1016/j.urology.2016.02.009.

[118] Bokarica P, Parazajder J, Mazuran B, Gilja I. Surgical treatment of Peyronie's disease based on penile length and degree of curvature. International Journal of Impotence Research 2005;17:170-4. https://doi.org/10.1038/sj.ijir.3901255.

[119] Langston JP, Carson CC. Peyronie Disease: Plication or Grafting. Urologic Clinics of North America 2011;38:207-16. https://doi.org/10.1016/j.ucl.2011.03.001.

[120] Ferretti L, Faix A, Droupy S. [Lapeyronie's disease]. Prog Urol 2013;23:674-84. https://doi.org/10.1016/j.purol.2013.02.012.

[121] Nesbit RM. Congenital curvature of the phallus: report of three cases with description of corrective operation. The Journal of Urology 1965;93:230-232.

[122] Pryor JP, Fitzpatrick JM. A new approach to the correction of the penile deformity in Peyronie's disease. J Urol 1979;122:622-3.

[123] Syed AH, Abbasi Z, Hargreave TB. Nesbit procedure for disabling Peyronie's curvature: a median follow-up of 84 months. Urology 2003;61:999-1003.

https://doi.org/10.1016/S0090-4295(02)02549-9.

[124] Essed E, Schroeder FH. New surgical treatment for Peyronie disease. Urology 1985;25:582-587.

[125] Ralph DJ, al-Akraa M, Pryor JP. The Nesbit operation for Peyronie's disease: 16-year experience. J Urol 1995;154:1362-3.

[126] Lopes I, Tomada N, Vendeira P. Penile corporoplasty with Yachia's technique for Peyronie's disease: Single center experience with 117 patients. Urol Ann 2013;5:167-71. https://doi.org/10.4103/0974-7796.115736.

[127] Kadioglu A, Sanli O, Akman T, Cakan M, Erol B, Mamadov F. Surgical Treatment of Peyronie's Disease: A Single Center Experience with 145 Patients. European Urology 2008;53:432-40. https://doi.org/10.1016/j.eururo.2007.04.045.

[128] Lemberger RJ, Bishop MC, Bates CP. Nesbit's operation for Peyronie's disease. BJU International 1984;56:721-723.

[129] Rehman J, Benet A, Minsky LS, Melman A. Results of surgical treatment for abnormal penile curvature: Peyronie's disease and congenital deviation by modified Nesbit plication (tunical shaving and plication). J Urol 1997;157:1288-91.

[130] Greenfield JM, Lucas S, Levine LA. Factors Affecting the Loss of Length Associated With Tunica Albuginea Plication for Correction of Penile Curvature. The Journal of Urology 2006;175:238-41. https://doi.org/10.1016/S0022-5347(05)00063-7.

[131] Savoca G, Scieri F, Pietropaolo F, Garaffa G, Belgrano E. Straightening Corporoplasty for Peyronie's Disease: A Review of 218 Patients with Median Follow-Up of 89 Months. European Urology 2004;46:610-4. https://doi.org/10.1016/j.eururo.2004.04.027.

[132] Schneider T, Sperling H, Schenck M, Schneider U, Rübben H. Treatment of penile curvature-how to combine the advantages of simple plication and the Nesbit-procedure by superficial excision of the tunica albuginea. World Journal of Urology 2003;20:350-355. 
[133] Kim DH, Lesser TF, Aboseif SR. Subjective Patient-Reported Experiences After Surgery for Peyronie's Disease: Corporeal Plication Versus Plaque Incision with Vein Graft. Urology 2008;71:698-702. https://doi.org/10.1016/j.urology.2007.11.065.

[134] Seveso M, Melegari S, De Francesco O, Macchi A, Romero Otero J, Taverna G, et al. Surgical correction of Peyronie's disease via tunica albuginea plication: long-term follow-up. Andrology 2018;6:47-52. https://doi.org/10.1111/andr.12431.

[135] Garaffa G, Sacca A, Christopher AN, Ralph DJ. Circumcision is not mandatory in penile surgery. BJU International 2010;105:222-4. https://doi.org/10.1111/j.1464410X.2009.08763.x.

[136] Kalsi J, Minhas S, Christopher N, Ralph D. The results of plaque incision and venous grafting (Lue procedure) to correct the penile deformity of Peyronie's disease. BJU Int 2005;95:1029-33. https://doi.org/10.1111/j.1464-410X.2005.05459.x.

[137] Dalkin BL, Carter MF. Venogenic impotence following dermal graft repair for Peyronie's disease. J Urol 1991;146:849-51. https://doi.org/10.1016/s0022-5347(17)37941-7. [138] Kadioglu A, Sanli O, Akman T, Ersay A, Guven S, Mammadov F. Graft materials in Peyronie's disease surgery: a comprehensive review. J Sex Med 2007;4:581-95. https://doi.org/10.1111/j.1743-6109.2007.00461.x.

[139] Chung E, Clendinning E, Lessard L, Brock G. Five-year follow-up of Peyronie's graft surgery: outcomes and patient satisfaction. J Sex Med 2011;8:594-600.

https://doi.org/10.1111/j.1743-6109.2010.02102.x.

[140] Garcia-Gomez B, Ralph D, Levine L, Moncada-Iribarren I, Djinovic R, Albersen M, et al. Grafts for Peyronie's disease: a comprehensive review. Andrology 2018;6:117-26. https://doi.org/10.1111/andr.12421.

[141] Levine LA, Burnett AL. Standard operating procedures for Peyronie's disease. J Sex Med 2013;10:230-44. https://doi.org/10.1111/j.1743-6109.2012.03003.x.

[142] Flores S, Choi J, Alex B, Mulhall JP. Erectile dysfunction after plaque incision and grafting: short-term assessment of incidence and predictors. J Sex Med 2011;8:2031-7. https://doi.org/10.1111/j.1743-6109.2011.02299.x.

[143] Rybak J, Papagiannopoulos D, Levine L. A retrospective comparative study of traction therapy vs. no traction following tunica albuginea plication or partial excision and grafting for Peyronie's disease: measured lengths and patient perceptions. J Sex Med 2012;9:2396-403. https://doi.org/10.1111/j.1743-6109.2012.02849.x.

[144] Devine CJ Jr, Horton CE. Surgical treatment of Peyronie's disease with a dermal graff. J Urol 1974;111:44-9.

[145] Hsu G-L, Chen H-S, Hsieh C-H, Chen RM, Wen H-S, Liu L-J, et al. Long-term results of autologous venous grafts for penile morphological reconstruction. J Androl 2007;28:18693. https://doi.org/10.2164/jandrol.106.000760.

[146] Ralph DJ. The surgical treatment of Peyronie's disease. Eur Urol 2006;50:196-8. https://doi.org/10.1016/j.eururo.2006.05.016.

[147] Chun JL, McGregor A, Krishnan R, Carson CC. A comparison of dermal and cadaveric pericardial grafts in the modified Horton-Devine procedure for Peyronie's disease. $\mathrm{J}$ Urol 2001;166:185-8.

[148] Das S. Peyronie's disease: excision and autografting with tunica vaginalis. J Urol 1980;124:818-9.

[149] Cormio L, Zucchi A, Lorusso F, Selvaggio O, Fioretti F, Porena M, et al. Surgical treatment of Peyronie's disease by plaque incision and grafting with buccal mucosa. Eur Urol 2009;55:1469-75. https://doi.org/10.1016/j.eururo.2008.11.041.

[150] Breyer BN, Brant WO, Garcia MM, Bella AJ, Lue TF. Complications of porcine small intestine submucosa graft for Peyronie's disease. J Urol 2007;177:589-91.

https://doi.org/10.1016/j.juro.2006.09.051. 
[151] Knoll LD. Use of small intestinal submucosa graft for the surgical management of Peyronie's disease. J Urol 2007;178:2474-8; discussion 2478.

https://doi.org/10.1016/j.juro.2007.08.044.

[152] Hatzichristodoulou G. Evolution of the surgical sealing patch TachoSil® in Peyronie's disease reconstructive surgery: technique and contemporary literature review. World J Urol 2020;38:315-21. https://doi.org/10.1007/s00345-019-02792-w.

[153] de Sallmard G, Morel-Journel N, Sbizzera M, Hanquiez P, Ruffion A, Terrier J-E. [Plaque incision and Tachosil graft in Peyronie's Disease]. Prog Urol 2020;30:119-25. https://doi.org/10.1016/j.purol.2019.11.011.

[154] Mulhall J, Anderson M, Parker M. A surgical algorithm for men with combined Peyronie's disease and erectile dysfunction: functional and satisfaction outcomes. J Sex Med 2005;2:132-8. https://doi.org/10.1111/j.1743-6109.2005.20113.x.

[155] Rolle L, Falcone M, Ceruti C, Timpano M, Sedigh O, Ralph DJ, et al. A prospective multicentric international study on the surgical outcomes and patients' satisfaction rates of the "sliding" technique for end-stage Peyronie's disease with severe shortening of the penis and erectile dysfunction. BJU Int 2016;117:814-20. https://doi.org/10.1111/bju.13371.

[156] Wilson SK, Delk JR. A new treatment for Peyronie's disease: modeling the penis over an inflatable penile prosthesis. J Urol 1994;152:1121-3. https://doi.org/10.1016/s00225347(17)32519-3.

[157] Tsambarlis PN, Chaus F, Levine LA. Successful Placement of Penile Prostheses in Men With Severe Corporal Fibrosis Following Vacuum Therapy Protocol. J Sex Med 2017;14:44-6. https://doi.org/10.1016/j.jsxm.2016.11.304. 


\section{Questionnaire sur la maladie de La Peyronie}

\section{INSTRUCTIONS :}

Ce questionnaire a pour objectif d'identifier tout problème que vous pourriez avoir en matière d'érection ou de rapports sexuels avec pénétration vaginale. Certaines questions concernent les rapports sexuels avec pénétration vaginale avec une partenaire féminine, d'autres non.

Veuillez répondre à toutes les questions dans l'espace prévu à cet effet. Vos réponses resteront strictement confidentielles.

Si vous N'AVEZ PAS eu de rapports sexuels avec pénétration vaginale avec une partenaire féminine au cours des 3 derniers mois, veuillez cocher la case ci-dessous et NE PAS REMPLIR le reste de ce questionnaire.

$\square$ Non, je n'ai eu aucun rapport sexuel avec pénétration vaginale au cours des 3 derniers mois

Si vous AVEZ eu des rapports sexuels avec pénétration vaginale avec une partenaire féminine au cours des 3 derniers mois, veuillez cocher la case ci-dessous et REMPLIR le reste de ce questionnaire.

Oui, j'ai eu des rapports sexuels avec pénétration vaginale au cours des 3 derniers mois

Combien de fois, environ, avez-vous eu des rapports sexuels avec pénétration vaginale au cours des 3 derniers mois?

Nombre de fois

À quelle date avez-vous eu des rapports sexuels avec pénétration vaginale pour la dernière fois ? 
Les hommes atteints de la maladie de La Peyronie peuvent avoir des problèmes au cours de rapports sexuels avec pénétration vaginale.

Les questions ci-dessous concernent la gravité de tout problème que vous pourriez avoir au cours de rapports sexuels avec pénétration vaginale.

Pour chaque problème indiqué ci-dessous, veuillez entourer le chiffre qui décrit le mieux jusqu'à quel point ce problème a été grave LA DERNIĖRE FOIS QUE VOUS AVEZ EU DES RAPPORTS SEXUELS AVEC PÉNÉTRATION VAGINALE.

VEUILLEZ ENTOURER UN CHIFFRE POUR CHAQUE QUESTION

Si vous n'avez pas eu ce problème, entourez le « 0 "

\begin{tabular}{|l|c|c|c|c|c|}
\hline \multicolumn{1}{|c|}{ Problème } & Aucun & Léger & Modéré & Grave & Très grave \\
\hline $\begin{array}{l}\text { 1. Inquiétude à l'idée d'endommager } \\
\text { votre pénis pendant un rapport } \\
\text { sexuel avec pénétration vaginale }\end{array}$ & 0 & 1 & 2 & 3 & 4 \\
\hline $\begin{array}{l}\text { 2. Déviation ou perte totale de rigidité } \\
\text { de votre pénis au cours d'un } \\
\text { rapport sexuel avec pénétration } \\
\text { vaginale }\end{array}$ & 0 & 1 & 2 & 3 & 4 \\
\hline $\begin{array}{l}\text { 3. Difficulté pour introduire votre pénis } \\
\text { en érection dans le vagin de votre } \\
\text { partenaire }\end{array}$ & 0 & 1 & 2 & 3 & 4 \\
\hline $\begin{array}{l}\text { 4. Difficultés avec certaines positions } \\
\text { que vous aviez l'habitude } \\
\text { d'apprécier lors de vos rapports } \\
\text { sexuels avec pénétration vaginale }\end{array}$ & 0 & 1 & 2 & 3 & 4 \\
\hline $\begin{array}{l}\text { 5. Maladresse lors de l'exécution de } \\
\text { certaines positions que vous aviez } \\
\text { l'habitude d'apprécier lors de vos } \\
\text { rapports sexuels avec pénétration } \\
\text { vaginale }\end{array}$ & 0 & 1 & 2 & 3 & 4 \\
\hline $\begin{array}{l}\text { 6. Inconfort lors de l'exécution de } \\
\text { certaines positions que vous aviez } \\
\text { l'habitude d'apprécier lors de vos } \\
\text { rapports sexuels avec pénétration } \\
\text { vaginale }\end{array}$ & 0 & 1 & 2 & 3 & 4 \\
\hline
\end{tabular}


En pensant à votre dernière érection ou à la dernière fois que vous avez eu des rapports sexuels avec pénétration vaginale, veuillez indiquer le niveau de douleur ou d'inconfort ressenti.

\section{VEUILLEZ ENTOURER UN CHIFFRE POUR CHAQUE QUESTION}

Si vous n'avez ressenti aucune douleur ou inconfort, entourez le « 0 »

7. Au cours des DERNIĖRES 24 HEURES, quel niveau de douleur ou d'inconfort avez-vous ressenti dans votre pénis lorsqu'il n'était PAS en érection? Veuillez répondre en pensant uniquement aux DERNIĖRES 24 HEURES.

Aucune douleur

ou inconfort

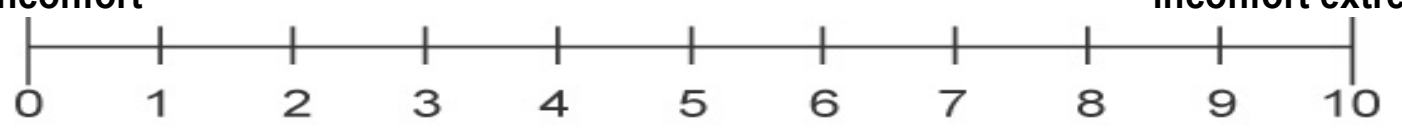

Douleur ou

8. En pensant à la DERNIĖRE FOIS où vous avez eu une érection, quel niveau de douleur ou d'inconfort avez-vous ressenti dans votre pénis lorsqu'il était en érection? Veuillez répondre en pensant à la DERNIÈRE FOIS OÙ VOUS AVEZ EU UNE ÉRECTION.

Aucune douleur

ou inconfort

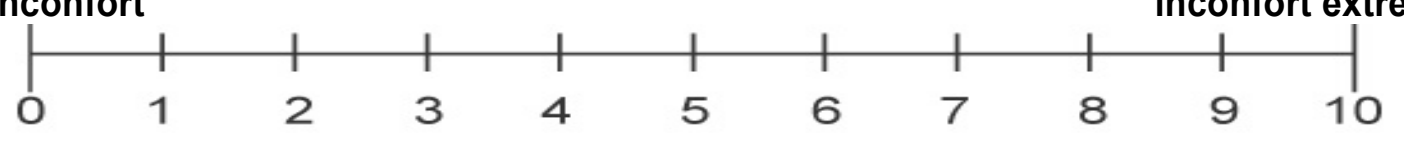

9. En pensant à la DERNIĖRE FOIS où vous avez eu des rapports sexuels avec pénétration vaginale, quel niveau de douleur ou d'inconfort avez-vous ressenti dans votre pénis lorsque vous avez eu ces rapports? Veuillez répondre en pensant à la DERNIÈRE FOIS OÙ VOUS AVEZ EU DES RAPPORTS SEXUELS AVEC PÉNÉTRATION VAGINALE.

Aucune douleur

Douleur ou ou inconfort

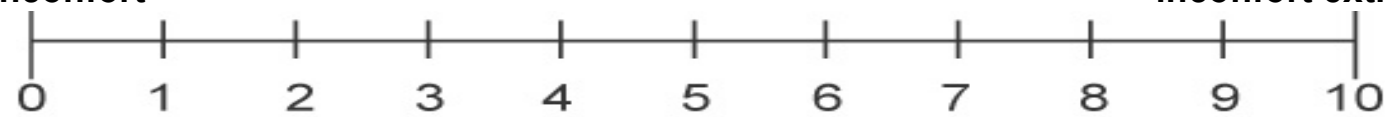


Les hommes atteints de la maladie de La Peyronie peuvent avoir des problèmes lors d'une érection et/ou de rapports sexuels avec pénétration vaginale. Ces problèmes peuvent être gênants pour certains hommes, et pour d'autres non.

\section{VEUILLEZ COCHER UNE CASE POUR CHAQUE QUESTION}

10. En pensant à la DERNIĖRE FOIS où vous avez eu une érection, dans quelle mesure avez-vous été gêné par une douleur ou un inconfort que vous avez peutêtre ressenti dans votre pénis en érection?

\section{Veuillez répondre en pensant à la DERNIÈRE FOIS OÙ VOUS AVEZ EU UNE \\ ÉRECTION.}

$\square \quad$ N'Al ressenti ni douleur ni inconfort d'aucune sorte - Veuillez passer à la question 11

Ai ressenti une douleur ou un inconfort et ai été :

$\square \quad$ Pas du tout gêné

$\square \quad$ Un peu gêné

$\square \quad$ Modérément gêné

$\square \quad$ Très gêné

$\square \quad$ Extrêmement gêné

11. En pensant à la DERNIĖRE FOIS où vous avez regardé votre pénis en érection, dans quelle mesure avez-vous été gêné par son apparence?

Veuillez répondre en pensant à la DERNIĖRE FOIS OÙ VOUS AVEZ EU UNE ÉRECTION.

$\square \quad$ Pas du tout gêné

$\square \quad$ Un peu gêné

$\square \quad$ Modérément gêné

$\square \quad$ Très gêné

$\square$ Extrêmement gêné 
12. Est-ce que votre maladie de La Peyronie rend vos rapports sexuels avec pénétration vaginale difficiles ou impossibles?

Non - Veuillez passer à la question 14

- Oui - Veuillez passer à la question 13

13. En pensant à la DERNIĖRE FOIS où vous avez eu ou tenté d'avoir des rapports sexuels avec pénétration vaginale, dans quelle mesure avez-vous été gêné par votre maladie de La Peyronie?

$\square$ Pas du tout gêné

$\square$ Un peu gêné

$\square$ Modérément gêné

Très gêné

Extrêmement gêné

14. Avez-vous des rapports sexuels avec pénétration vaginale MOINS SOUVENT qu'auparavant à cause de votre maladie de La Peyronie?

Non - Merci, vous avez terminé de remplir ce questionnaire

Oui - veuillez passer à la question 15

15. Dans quelle mesure êtes-VOUS gêné par le fait d'avoir moins souvent des rapports sexuels avec pénétration vaginale?

$\square$ Pas du tout gêné

$\square$ Un peu gêné

$\square$ Modérément gêné

$\square$ Très gêné

$\square$ Extrêmement gêné

Merci, vous avez terminé de remplir ce questionnaire. 


\section{SCORE IIEF5}

Ce questionnaire permet d'évaluer votre fonction sexuelle au court des 6 derniers mois :

\section{Au cours des six derniers mois :}

I. A quel point étiez-vous sûr de pouvoir avoir une érection et de la maintenir ?

1. Pas sûr du tout

2. Pas très sûr

3. Moyennement sûr

4. Sûr

5. Très sûr

II. Lorsque vous avez eu des érections à la suite de stimulations sexuelles, avec quelle fréquence votre pénis a-t-il été suffisamment rigide (dur) pour permettre la pénétration?

$0 . \quad$ Je n'ai pas été stimulé sexuellement

1. Presque jamais ou jamais

2. Rarement (beaucoup moins que la moitié du temps)

3. Quelquefois (environ la moitié du temps)

4. La plupart du temps (beaucoup plus que la moitié du temps)

5. Presque tout le temps ou tout le temps

III. Lorsque vous avez essayé d'avoir des rapports sexuels, avec quelle fréquence avez-vous pu rester en érection après avoir pénétré votre partenaire?

$0 . \quad$ Je n'ai pas essayé d'avoir de rapports sexuels

1. Presque jamais ou jamais

2. Rarement (beaucoup moins que la moitié du temps)

3. Quelquefois (environ la moitié du temps)

4. La plupart du temps (beaucoup plus que la moitié du temps)

5. Presque tout le temps ou tout le temps

IV. Pendant vos rapports sexuels, à quel point vous a-t-il été difficile de rester en érection jusqu'à la fin de ces rapports ?

0. Je n'ai pas essayé d'avoir de rapports sexuels

1. Extrêmement difficile

2. Très difficile

3. Difficile

4. Un peu difficile

5. Pas difficile

V. Lorsque vous avez essayé đavoir des rapports sexuels, avec quelle fréquence en avez-vous été satisfait?

0. Je n'ai pas essayé d'avoir de rapports sexuels

1. Presque jamais ou jamais

2. Rarement (beaucoup moins que la moitié du temps)

3. Quelquefois (environ la moitié du temps)

4. La plupart du temps (beaucoup plus que la moitié du temps)

5. Presque tout le temps ou tout le temps

Interprétation : :

Trouble de l'érection sévère (score de 5 à 10), modéré (1I à 15), léger (16 à 20), fonction érectile normale (21 à 25) et non interprétable ( 1 à 4 ). 


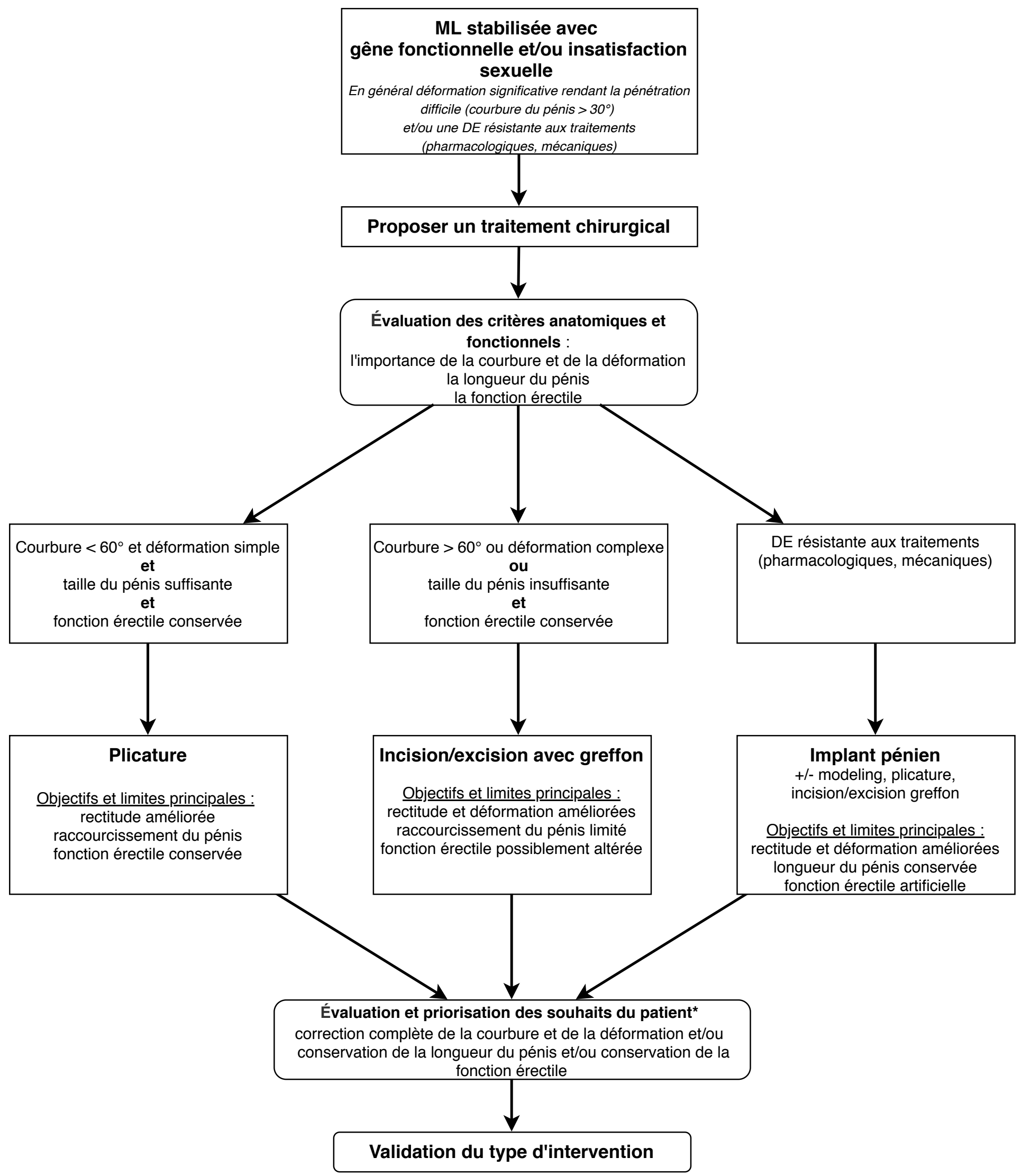

Figure 1. Algorithme de la prise en charge chirurgicale dans la maladie de Lapeyronie (ML)

* Une information claire et objective sur l'efficacité et les complications de l'intervention doit être délivrée au patient.

Ses attentes doivent être énoncées et des objectifs réalistes définis.

L'objectif principal de l'intervention est d'améliorer les difficultés de pénétration secondaires à la courbure, en conservant une érection rigide et une taille de pénis suffisante. La satisfaction post-opératoire est conditionnée par la compréhension des résultats attendus et ses limites. 


\section{Erection Hardness Score (EHS)}

Score de rigidité de l'érection

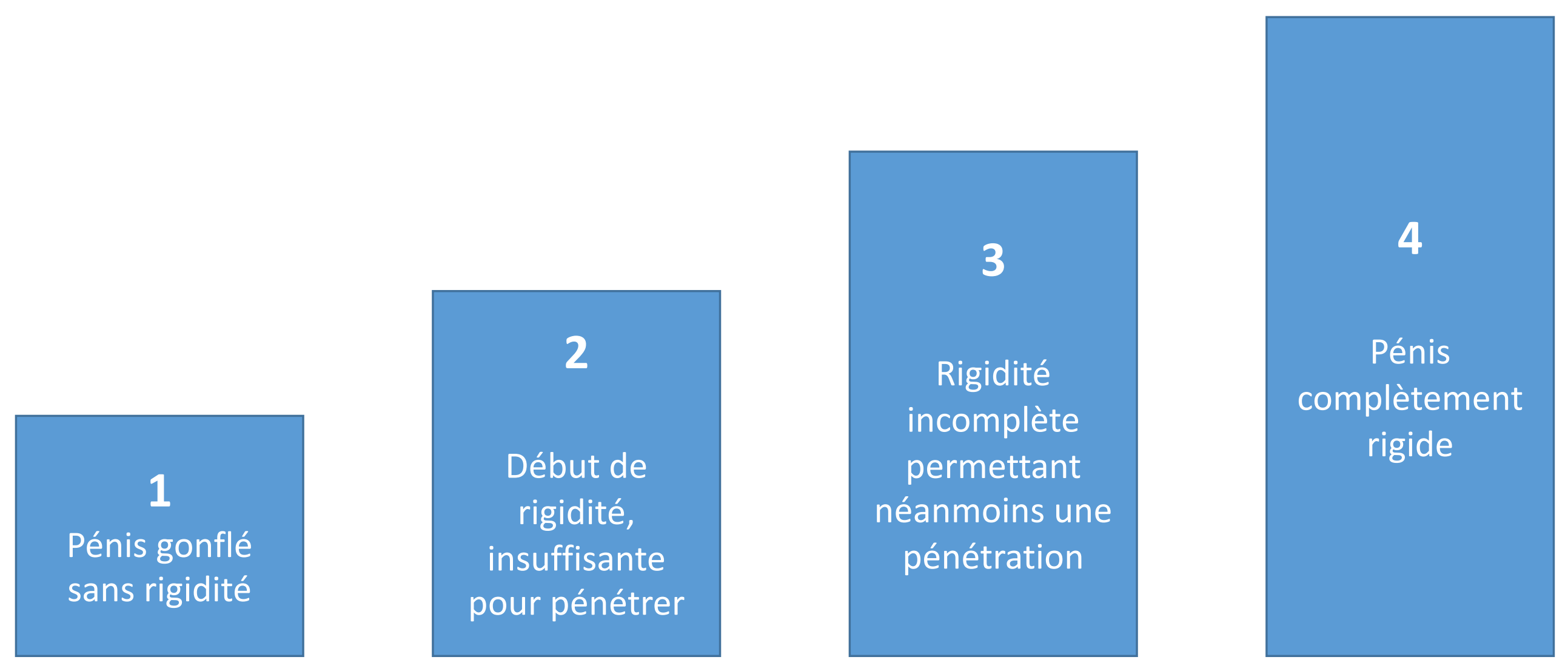

